\title{
Towards clinical application of pronuclear transfer to prevent mitochondrial DNA disease
}

\author{
Louise A. Hyslop ${ }^{1,2}$, Paul Blakeley ${ }^{3}$, Lyndsey Craven ${ }^{4}$, Jessica Richardson ${ }^{1}$, Norah M.E. \\ Fogarty $^{3}$, Elpida Fragouli ${ }^{5}$, Mahdi Lamb ${ }^{1}$, Sissy E. Wamaitha ${ }^{3}$, Nilendran Prathalingam ${ }^{1,2}$, \\ Qi Zhang ${ }^{1}$, Hannah O'Keefe ${ }^{1}$, Yuko Takeda ${ }^{1}$, Lucia Arizzi ${ }^{1,2}$, Samer Alfarawati ${ }^{5}$, Helen A. \\ Tuppen $^{4}$, Laura Irving ${ }^{1}$, Dimitrios Kalleas ${ }^{1}$, Meenakshi Choudhary ${ }^{2}$, Dagan Wells ${ }^{6}$, Alison $\mathbf{P}$ \\ Murdoch $^{2}$, Douglass M. Turnbull ${ }^{4}$, Kathy K. Niakan ${ }^{3}$, and Mary Herbert ${ }^{1,2}$ \\ ${ }^{1}$ Wellcome Trust Centre for Mitochondrial Research, Institute of Genetic Medicine, Biomedicine \\ West Wing, Centre for Life, Times Square, Newcastle upon Tyne, NE1 3BZ, UK \\ ${ }^{2}$ Newcastle Fertility Centre, Biomedicine West Wing, Centre for Life, Times Square, Newcastle \\ upon Tyne, NE1 4EP, UK \\ ${ }^{3}$ The Francis Crick Institute, Human Embryo and Stem Cell Laboratory, Mill Hill Laboratory Mill \\ Hill, London, NW7 1AA, UK \\ ${ }^{4}$ Wellcome Trust Centre for Mitochondrial Research, Institute of Neuroscience, Newcastle \\ University, The Medical School, Framlington Place, Newcastle upon Tyne, NE2 4HH, UK \\ ${ }^{5}$ Reprogenetics UK, Institute of Reproductive Sciences, Oxford Business Park North, Oxford OX4 \\ $2 \mathrm{HW}, \mathrm{UK}$ \\ ${ }^{6}$ University of Oxford, Nuffield Department of Obstetrics and Gynaecology, John Radcliffe \\ Hospital, Oxford OX3 9DU, UK
}

\begin{abstract}
Mitochondrial DNA (mtDNA) mutations are maternally inherited and are associated with a broad range of debilitating and fatal diseases 1 . Reproductive technologies designed to uncouple the inheritance of mtDNA from nuclear DNA may enable affected women to have a genetically related child with a greatly reduced risk of mtDNA disease. Here we report the first preclinical studies on pronuclear transplantation (PNT). Surprisingly, techniques used in proof of concept studies involving abnormally fertilized human zygotes 2 were not well tolerated by normally fertilized zygotes. We have therefore developed an alternative approach based on transplanting
\end{abstract}

Users may view, print, copy, and download text and data-mine the content in such documents, for the purposes of academic research, subject always to the full Conditions of use:http://www.nature.com/authors/editorial_policies/license.html\#terms

Author contributions

M.H. and L.A.H. conceived and designed the PNT experiments. L.A.H, L.I., L.C. and L.A. performed PNT experiments and embryo manipulations. J.R., D.K. and Q.Z. performed cell counts. D.W., E.F. and S.A. performed whole genome amplification and arrayCGH. K.K.N., P.B. and N.M.E.F. performed RNA-seq experiments. L.C., H.A.T. and D.M.T. measured mtDNA carryover and performed mtDNA haplogroup analysis. N.P., K.K.N, N.M.E.F, S.E.W., Y.T., and H.O'K. derived, cultured and characterized ESC lines. K.K.N., P.B., M.L., J.R., L.A.H, L.C., Y.T. P.B. and M.H. analyzed data. A.P.M. and M.C. coordinated the oocyte donation program. M.H. wrote the manuscript with input from D.M.T., D.W., K.K.N., J.R. and M.L.

Competing interests

The authors declare no competing financial interests. 
pronuclei shortly after completion of meiosis rather than shortly before the first mitotic division. This promotes efficient development to the blastocyst stage with no detectable effect on aneuploidy or gene expression. Following optimisation, mtDNA carryover was reduced to $<2 \%$ in the majority (79\%) of PNT blastocysts. The importance of reducing carryover to the lowest possible levels is highlighted by a progressive increase in heteroplasmy in a stem cell line derived from a PNT blastocyst with 4\% mtDNA carryover. We conclude that PNT has the potential to reduce the risk of mtDNA disease, but it may not guarantee prevention.

Predicting the risk of serious disease in children of women who carry mtDNA mutations is complicated by a number of factors. Mutations in mtDNA can be either homoplasmic (all copies of mtDNA are mutated) or heteroplasmic (mixture of mutated and wild-type mtDNA). In the case of heteroplasmy, women produce oocytes with widely varying mutation loads3. While pathogenicity is generally proportional to the ratio of mutated to wild-type mtDNA, the severity of disease for a given mutation load can vary, even amongst homoplasmic individuals4. The resulting unpredictability in the risk of transmitting disease raises profoundly difficult reproductive decisions for women from affected families. While preimplantation genetic diagnosis (PGD) can be used to reduce the risk of mtDNA disease by identifying embryos with low mutation loads5, it is not useful for women who are homoplasmic for pathogenic mtDNA mutations6. In such cases, it may be possible to reduce the risk of transmission by transplanting the oocyte nuclear DNA to an enucleated donor oocyte free of pathogenic mtDNA mutations.

Progression through female meiosis offers a number of opportunities for transplanting nuclear DNA. Proof of concept studies in human7,8, indicate that transplantation of the nuclear genome between oocytes arrested at metaphase of meiosis II (MII) is associated with a high incidence of abnormal fertilization7. An alternative approach is to transplant the nuclear genome after fertilization, when the haploid maternal and paternal genomes are separately packaged in large clearly visible pronuclei. First performed in mouse zygotes more than three decades ago9, pronuclear transfer (PNT) is typically performed during the G2 phase of the $1^{\text {st }}$ mitotic cell cycle. Using this approach, we have previously demonstrated that PNT between abnormally fertilized human zygotes is technically feasible2. However, their limited capacity for onward development has been a major barrier to further investigation of the therapeutic potential of PNT.

Here we investigate the effect of PNT on normally fertilized human zygotes. We found that the procedures (Extended Data Fig. 1 a,b) previously used for abnormally fertilized zygotes2 resulted in reduced survival. Because developmental competence is correlated with accelerated division to the 2-cell stage10, we asked whether the timing of PNT might be too close to onset of $1^{\text {st }}$ mitosis in normally fertilized zygotes (Fig. 1a). To address this, we undertook a series of experiments in which the PN were transplanted shortly after they first appear ( $\sim \mathrm{hr}$ after insemination; Fig. 1b, Videos 1 and 2). Initially, we added sucrose to the enucleation medium to facilitate enucleation and fusion by inducing shrinkage of the cytoplasm (Fig. 1b). However, this was later abandoned to reduce the karyoplast mtDNA content and had minimal effect on survival (see below). Our data indicate that early PNT (ePNT) promotes survival (92\% vs 59\% for late PNT (LtPNT); $P<0.01$; Fig. 1c). Moreover, 
ePNT zygotes showed normal PN abuttal and division to the 2-cell stage (Extended Data Fig. $1 \mathrm{c}, \mathrm{d})$, indicating that sperm centriole function was not disrupted 11.

Blastocyst formation, which is essential for implantation, is marked by allocation of cells to the inner cell mass (ICM), or to an outer layer of trophectoderm (TE) cells12 at 5-6 days after fertilisation in vitro. The morphology of the ICM and TE correlates well with implantation and is used to assess blastocyst quality in clinical IVF programmes (Extended Data Fig. 2a-d). While the increased survival of ePNT zygotes (Series I) resulted in improved blastocyst formation compared with LtPNT, both approaches produced few good quality blastocysts (Extended Data Fig. 2e,f). Control experiments in which PN were replaced in the same zygote (autologous ePNT) indicated that blastocyst quality was compromised by the manipulations (Fig. 2a and Extended Data Fig. 2f). To address this we modified the manipulation medium, removing $\mathrm{Ca}^{2+}$ and $\mathrm{Mg}^{2+}$ and reducing the $\mathrm{HVJ}-\mathrm{E}$ concentration tenfold8. In addition, we switched from a two-step to a single-step culture medium, in which embryos remained for the duration of culture. Under these conditions (ePNT Series II), blastocyst formation and quality did not differ between unmanipulated controls and technical controls (Fig. 2a,b). Similarly, heterologous ePNT, which involved reciprocal transfers between zygotes from fresh and vitrified oocytes, had no detectable effect on blastocyst quality (Fig. 2b,c). Consistent with the improved quality, nuclear counts indicated that ePNT blastocyst cell numbers were equivalent to controls (Extended Data Fig. 2g,h). However, heterologous PNT resulted in reduced blastocyst formation (Fig. 2b), possibly due to an effect of vitrification at the MII stage13, which was not ameliorated by delaying vitrification until after exit from MII (Extended Data Fig. 3a-f).

Analysis of aneuploidy by array-based comparative genomic hybridization (array-CGH) indicated that while the majority of poor quality ePNT blastocysts were aneuploid for multiple chromosomes (Fig. 2d and Extended Data Fig. 4), the overall incidence of aneuploidy was comparable between ePNT and control blastocysts, and was similar to a reference dataset of IVF blastocysts, in which female age was matched to the karyoplast donors (Fig. 2e). These data indicate that the ePNT procedure does not result in an increased incidence of aneuploid blastocysts.

We next determined whether ePNT alters the pattern of gene expression in human blastocysts by performing RNA sequencing (RNA-seq) on single cells microdissected from ePNT and control blastocysts (Extended Data Fig. 5a, b). For reference, we also included a previously published series of unmanipulated blastocysts 14 . The ePNT blastocysts included in these experiments were generated by fusion of cytoplasts and karyoplasts with the same (autologous and homologous ePNT), or different (heterologous ePNT) mitochondrial genotypes (Extended Data Fig. 5b).

To test for differences in global gene expression, we performed principal component analysis (PCA) on normalized RNA-seq data (Extended Data Fig. 5c). We first determined whether PCA is sufficiently sensitive to detect differences in global gene expression between good and poor quality blastocysts. Plotting PC1 against PC2, which together account for the largest contributions to variation in global gene expression, revealed a high proportion of outliers among samples from poor quality blastocysts (Fig. 3a,b). By contrast, samples from 
good quality ePNT blastocysts clustered closely with controls (Fig. 3c). To determine whether additional principal components, however minor, might distinguish differences between good quality ePNT and control blastocysts, we plotted all combinations of the first 10 principal components. In each combination we found that ePNT samples cluster with controls (Extended Data Fig. 6a). Furthermore, we were able to distinguish distinct populations of cells corresponding to the three cell lineages of the mammalian blastocyst 14 (Extended Data Fig. 6b). This was confirmed by t-SNE, a non-linear method for dimensionality reduction15 (Extended Data Fig. 6c). Consistent with this, unsupervised hierarchical clustering revealed that ePNT and control samples cluster together based on lineage (Extended Data Fig. 7a). Together these findings indicate that single cell RNA-seq reliably detects differences in gene expression, and that global and lineage-associated gene expression is indistinguishable between control and ePNT blastocysts.

To address the question of whether ePNT specifically affects expression of mtDNA-encoded OXPHOS genes, we generated a heatmap after unsupervised hierarchical clustering. This revealed wide variation in the level of mtDNA OXPHOS gene expression within and between ePNT and control blastocysts. However, samples from both groups clustered together irrespective of whether the karyoplast and cytoplast contained the same, or different, mitochondrial genomes (Extended Data Fig. 7b). This suggests that switching nuclear genomes does not alter mitochondrial gene expression.

Based on evidence from a range of pathogenic mutations, the probability of developing or transmitting disease is low when mutation loads are $<18 \% 16$ and $<5 \% 17$ respectively. Thus, reducing the contribution of karyoplast mtDNA to $<5 \%$ has the potential to prevent transmission to subsequent generations. The level of mtDNA carryover during PN transfer was measured by pyrosequencing (Extended Data Fig. 8a-c) using clumps of cells from day 6 ePNT blastocysts $(n=40)$ generated by reciprocal transfer between zygotes from fresh and vitrified oocytes (Fig. 4a). Despite removal of excess cytoplasm before karyoplast fusion (Video 2 and Fig. 1b), we found that heteroplasmy was $>5 \%$ in a high proportion (28\%) of samples enucleated in the presence of sucrose. This was significantly reduced by omission of sucrose (Fig. 4b and Extended Data Fig. 8d), most likely due to an osmotic effect. MtDNA carryover was further reduced in blastocysts whose cytoplasts originated from freshly harvested rather than vitrified oocytes (Fig. 4c), which may be explained by a high incidence of cytoplasmic leakage from the latter. Thus, the efficacy of ePNT in preventing mtDNA disease is likely to be increased by vitrifying patient rather than donor oocytes. Based on our findings, this approach results in $<2 \%$ heteroplasmy in the majority (79\%) of blastocysts and none with $>5 \%$ heteroplasmy (Extended Data Fig. 8e,f). Notably, those with $>2 \%$ heteroplasmy were predicted by technical problems such as leakage from the cytoplast or inadequate shearing of cytoplasm from the karyoplast. Such factors could be taken into account when selecting embryos for use in clinical treatment.

To assess the potential fate of karyoplast mtDNA under conditions where it can replicate18, we derived hESC lines ( $n=5$ ) from ePNT blastocysts (Extended Data Fig. 9a-f). While all PNT-hESC lines showed low levels of heteroplasmy at passage 1 (P1), one line (36PNThESC), derived from a blastocyst with $4 \%$ mtDNA carryover, showed an upward drift with wide variation in heteroplasmy between colonies by P12 (Fig. 4d). This was confirmed by 
experiments in which individual colonies were subcloned and cultured for multiple passages (Extended Data Fig. 10). Interestingly, the karyoplast and cytoplast donors for 36PNT belonged to the same mtDNA haplogroup (Extended Data Fig. 9f), however, we cannot exclude the possibility that sequence variants in the karyoplast donor mtDNA might have conferred a replicative advantage 19. While the biological basis remains to be established, the relevance of the increased heteroplasmy to development in vivo is unclear. For example, recent reports indicate that pluripotent cells derived from heteroplasmic fibroblasts exhibit a bimodal drift towards homoplasmy, which is not observed in the parental lines20,21. Moreover, with the exception of one controversial case22,23, a number of reports24,25, together with our own unpublished data, indicate that levels of heteroplasmy in preimplantation embryos mirrors that in babies born following PGD. Nonetheless, the finding underscores the importance of reducing mtDNA carryover to the lowest possible levels and suggests that guaranteed prevention of disease will depend on complete elimination of karyoplast mtDNA.

In conclusion, the work reported here represents a significant advance towards understanding the therapeutic potential of PNT in preventing transmission of mtDNA disease. Transplanting the PN shortly after completion of meiosis resulted in improved survival. Further optimization of enucleation and embryo culture procedures promoted development of good quality blastocysts whose gene expression and incidence of aneuploidy did not differ from controls. Our findings also indicate that vitrification of patient rather than donor oocytes will likely minimize mtDNA carryover. This offers the added advantage of stockpiling patient oocytes before they become susceptible to age-related meiotic aneuploidy26. Given the low levels of mtDNA carryover using optimised procedures, we believe that ePNT has the potential to reduce risk of mtDNA disease. However, until more is known about the post-implantation fate of karyoplast mtDNA, it should be considered in combination with prenatal screening.

\section{Methods}

\section{Human oocytes and manipulations}

The study was approved by the Newcastle and North Tyneside Research Ethics Committee and was licensed by the UK Human Fertilisation and Embryology Authority (HFEA). Informed consent was obtained from all donors by research nurses who were not directly involved in the research, or in the clinical treatments of women participating in the study. Human oocytes $(n=523)$ included in this study were donated either by women undergoing infertility treatment ( $n=44$ oocytes from 6 donors, age range 25-36 years) as part of an "egg sharing" programme27, or by non-patient donors ( $n=479$ oocytes from 57 donors, age range 21-36 years). Donors were compensated in accordance with current HFEA guidance on payments for donors28. Non-patient donors received financial compensation of $£ 500$ per donation cycle. Compensation under the "egg share" programme consisted of a subsidy $(£ 1,500)$ from research funds towards the cost of treatment for self-funded patients27, or an additional fully funded treatment cycle for those who did not become pregnant following NHS-funded treatment. 
Oocytes were collected by ultrasound-guided follicle aspiration and the surrounding cumulus cells were removed using hyaluronidase (HYASE, Vitrolife). MII oocytes were identified by the presence of the $1^{\text {st }}$ polar body and were fertilised by intracytoplasmic sperm injection (ICSI) using sperm donated specifically for this project.

\section{Oocyte Vitrification}

MII oocytes were either vitrified or used immediately for PNT experiments. The majority ( $n=107)$ of vitrified oocytes were vitrified at the MII stage. We also conducted a series of experiments in which vitrification was performed following completion of MII (at the 2PB stage; $\sim 5.5$ hours post ICSI; $n=34$ ), to determine whether blastocyst development might be improved. Vitrification and warming were performed using the RapidVit and RapidWarm oocyte kits (Vitrolife, Sweden). Oocytes were stored in liquid nitrogen until required.

\section{Pronuclear Transfer (PNT)}

PNT was performed either at 16-20 hours after ICSI (Late PNT; LtPNT), or at 8-10 hours after ICSI (early PNT; ePNT). In the case of ePNT, two main series of experiments (Series I and Series II) were performed. A total of 51 zygotes from 10 donors were used for LtPNT ( $n=12$ controls; $n=39$ LtPNT). For ePNT experiments we used 58 zygotes from 13 donors in Series I ( $n=19$ controls; $n=39$ ePNT, and 131 zygotes from 30 donors in Series II ( $n=30$ controls; $n=101$ ePNT). Thirty-four zygotes from 13 donors were used for ePNT experiments involving oocytes vitrified at the $2 \mathrm{~PB}$ stage.

Two types of PNT experiments were conducted: (1) Autologous (Atgl) PNT, which involved removal and replacement of $\mathrm{PN}$ in the same zygote was performed to distinguish between technical and biological effects. (2) Heterologous (Het) PNT involved reciprocal transfer between pairs of zygotes, either from the same of different donors. Heterologous PNT between zygotes from different donors involved reciprocal transfer between zygotes originating from fresh and vitrified oocytes. This gave rise to reconstituted zygotes consisting of cytoplasts from fresh oocytes and karyoplasts from vitrified oocytes, or vice versa. These combinations are termed FreshCy and VitCy respectively (see Extended Data Fig. 4a). In one set of ePNT experiments, which gave rise to a single ePNT blastocyst, the two donors were sisters and therefore have the same mitochondrial genotype. For the purpose of the gene expression experiments, these are referred to as homologous transfers.

The PNT procedure was performed in an isolator-based workstation (Vitrosafe Ltd) with temperature, $\mathrm{CO}_{2}$ and $\mathrm{O}_{2}$ control29 containing an inverted microscope (TE2000-U, Nikon) fitted with micromanipulators (Integra Ti, Research Instruments) and a laser objective (Saturn Active, Research Instruments). PNT procedures took $\sim 15 \mathrm{~min}$ to complete and involved the following steps: (1) Zygotes in which 2PN were visible were placed in enucleation medium with cytoskeletal inhibitors. In all cases, nocodazole $(10 \mu \mathrm{g} / \mathrm{ml})$ was used to depolymerise microtubules. In LtPNT experiments we used either cytochalasin B $(5 \mu \mathrm{g} / \mathrm{ml})$ or Latrunculin A $(2.5 \mu \mathrm{M}$ or $5 \mu \mathrm{M})$ to disable the actin cytoskeleton. We subsequently used Latrunculin A $(2.5 \mu \mathrm{M})$ for all ePNT experiments. For LtPNT and ePNT (series I) experiments, enucleation was performed in G-1 Plus medium (Vitrolife, Sweden). We used Sydney IVF Embryo Biopsy Medium (Cook Medical), which does not contain 
$\mathrm{Ca}^{2+}$ and $\mathrm{Mg}^{2+}$ for ePNT (Series II) experiments. Enucleation was performed in the presence or absence of sucrose $(0.125 \mu \mathrm{m})$. Addition of sucrose increased the osmolarity of the enucleation medium from $280 \mathrm{mosm} / \mathrm{L}$ to $449 \mathrm{mosm} / \mathrm{L}$, which induced shrinkage of the cytoplasm, thereby facilitating enucleation. (2) A laser objective (Saturn Active, Research Instruments) was used to create an opening in the zona pellucida for insertion of the enucleation/fusion pipette. The inner diameter pipette measurements were $25-35 \mu \mathrm{m}$ for LtPNT, and $17 \mu \mathrm{m}$ for ePNT. (3) The PN, surrounded by a small amount of cytoplasm, were aspirated into the enucleation pipette, either as a single karyoplast, or as two separate karyoplasts (see Videos 1 and 2). (4) Karyoplasts were briefly exposed to a suspension of HVJ-E; GenomONE-CF Ex (Cosmo Bio, Japan). Undiluted suspension was used for LtPNT and ePNT (Series I) and a 1:10 dilution was used for ePNT (Series II). (5) The pipette containing the karyoplasts was inserted through the laser-drilled opening in the zona pellucida and karyoplasts were gently expelled into the perivitelline space and allowed to fuse with the cytoplast. (6) Reconstituted and control zygotes were cultured either in a sequential medium G-1 Plus (day 1-3)/ G-2 Plus (day 3-6) (Vitrolife; LtPNT and ePNT (series I)), or in the single-step G-TL medium (Vitrolife; ePNT (series II)) from day 1-6. Time-lapse embryo imaging was performed for three sets of ePNT (Series II) experiments using the Primo Vision Time-lapse monitoring system (Vitrolife, Sweden).

\section{Overview of experiments on PNT zygotes}

Survival of reconstituted zygotes was initially compared between LtPNT and ePNT (Series I) and was subsequently recorded for all ePNT zygotes. In ePNT (Series II), the first mitotic division was monitored by time-lapse imaging in two experiments. All zygotes submitted to PNT were included in the analysis of development to the blastocyst stage. Zygotes (controls and PNT) that developed to the blastocyst stage were graded and included in the analysis of blastocyst quality. Blastocyst formation and grade were assessed on day 6 for LtPNT, and on days 5 and 6 for ePNT, except in the case of two ePNT (Series II) experiments, which were assessed only on day 6 . These experiments are not included in the day 5 analysis shown in Fig. 2b and in Extended Data Fig. 3d-f.

Blastocyst cell counts were performed primarily to gain insight into the causes of poor blastocyst quality in the LtPNT and ePNT (Series I). Data on blastocyst cell counts were obtained from LtPNT ( $n=6)$ and ePNT: Series I $(n=8)$ and Series II $(n=5)$. Further analyses, including aneuploidy, gene expression, mtDNA carryover, and $\mathrm{hESC}$ derivation were conducted on Series II blastocysts only. Where possible, we performed multiple investigations on individual blastocysts. In accordance with our Local Research Ethics Committee approval and HFEA licence, these were performed on day 6. Unmanipulated control blastocysts and ePNT blastocysts were used for aneuploidy and gene expression, or aneuploidy and hESC derivation. The blastocyst grades shown for each of these analyses refer to the grades on day 6. The numbers of blastocysts used for each set of experiment were: Aneuploidy screening (PNT: $n=30$ from 20 donors; Control: $n=11$ from 10 donors), gene expression analysis (PNT: $n=11$ from 10 donors; Control: $n=3$ from 3 donors), mtDNA carryover (PNT: $n=40$ from 28 donors), hESC derivation (PNT: $n=15$ from 13 donors; Control: $n=6$ from 4 donors). 


\section{Embryo grading}

Embryos were graded using the UK National External Quality Assessment Service (NEQAS) grading schemes for embryos and blastocysts30. Blastocysts were assigned a three digit grade representing a score of: 1-6 for the extent of blastocoel expansion, 1-5 for the inner cell mass appearance and 1-3 for the trophectoderm appearance31. The grade was converted to a quality category using the table in Extended Data Fig. 2c.

\section{Blastocyst Nuclear Counts}

Day 6 blastocysts were fixed using 4\% PFA at $\mathrm{pH}$ 7.4. Nuclear staining was carried out using DAPI (Vectashield). Blastocysts were imaged using an inverted confocal microscope (Nikon AIR, Japan) with a 20X objective (Plan Apo, Nikon) and NIS-elements image software. Z-steps were taken at $\sim 1 \mu \mathrm{m}$ intervals and nuclear counts performed using ImageJ software.

\section{Aneuploidy screening}

Clumps of cells were harvested from ePNT blastocysts for whole genome amplification followed by microarray-CGH analysis according to a previously validated protocol using 24Sure Cytochip (Illumina, Cambridge, UK). Cells were obtained from the TE, ICM or both. Lysis and whole genome amplification was performed using the SurePlex kit (Illumina) according to the manufacturer's instructions. Samples from ePNT blastocysts were labelled with Cy3 while a commercially available reference 46,XY DNA was labelled with Cy5 (Illumina)32. A laser scanner (InnoScan 710, Innopsys, Carbonne, France) was used to analyse the microarrays after washing and drying. The resulting images were analysed using BlueFuse Multi analysis software (Illumina).

\section{Gene expression analysis by single cell RNA-seq}

\section{Blastocyst disaggregation}

Blastocyst disaggregation was performed using an Olympus IX73 microscope and a Saturn 5 laser (Research Instruments) as described previously 14. Embryos were placed in drops of GMOPS solution (Vitrolife, Sweden) on a petri dish overlaid with mineral oil for micromanipulation. The separated ICM and polar TE were washed in $\mathrm{Ca}^{2+}$ and $\mathrm{Mg}^{2+}$-free PBS (Invitrogen) and incubated in 0.05\% trypsin/EDTA (Invitrogen) for 5-10 minutes. Trypsin was quenched using Global Media supplemented with $5 \mathrm{mg} / \mathrm{mL}$ LifeGlobal Protein Supplement. Single cells were isolated using a $30 \mu \mathrm{m}$ inner diameter blastomere biopsy pipette (Research Instruments).

\section{cDNA synthesis and amplification}

cDNA was synthesised using SMARTer Ultra Low Input RNA for Illumina Sequencing-HV kit (Clontech Laboratories, Inc.) according to the manufacturer's guidelines and as previously published14. cDNA was sheared using Covaris $\mathrm{S} 2$ with the modified settings 10\% Duty, Intensity 5, Burst Cycle 200 for 2 min. Libraries were prepared using Low Input Library Prep Kit (Clontech Laboratories, Inc.) according to manufacturer's instructions. Library quality was assessed with an Agilent 2100 BioAnalyser and concentration measured 
with a Qubit 2.0 Fluorometer (Life Technologies UK Ltd.). Libraries were submitted for 50bp paired-end sequencing using standard Illumina adapters on Illumina HiSeq 2000. Samples with primer contamination and amplification bias, identified by an unequal proportion of ATGC nucleotide percentages, were excluded from subsequent analysis.

\section{RNA-seq data analysis}

The quality of the RNA-seq data was evaluated using the FastQC tool. Reads were aligned to the human genome sequence hg 19 using Tophat233, and samples with low percentage mapping ( $<50 \%)$ were excluded from subsequent analysis. The number of reads mapping uniquely to each gene was counted using the program htseq-count 34 . The individual count files for each sample were normalised using both the RPKM function in the edgeR package 35 and a variance-stabilizing transformation from the DESeq2 package36.

A principal component analysis (PCA) of the top 12,000 most variably expressed genes was performed blind to sample origin on all ePNT and control samples to investigate differences in global gene expression. The PCA was generated using the R package prcomp, using both the scaling and centering options. A subsequent PCA excluded samples below grade C, which were generally aneuploid for multiple chromosomes. An R script was used to perform unsupervised hierarchical clustering and to generate a heatmap using the pheatmap $\mathrm{R}$ package. An alternative approach for data dimensionality reduction was performed using the t-SNE algorithm. The top 5 principal components of the VST-normalised count data were used as input for the R implementation of t-SNE. DESeq2 was applied to the read counts for the ePNT and control data to identify differential expressed genes in the primitive endoderm, trophectoderm and epiblast samples. Raw data and an RPKM table have been deposited into Gene Expression Omnibus (GSE76284).

\section{MtDNA carryover analysis}

\section{MtDNA extraction and mtDNA sequencing}

The control region of the mitochondrial genome from oocyte donors was sequenced using ovarian follicular cells harvested at the time of oocyte retrieval, or cumulus cells removed from the oocyte in preparation for ICSI. DNA extraction from follicular cells was performed using the QIAamp ${ }^{\circledR}$ DNA Mini kit according to the manufacturer's instructions (Qiagen). Cumulus cells were lysed for 2 hours in a lysis buffer $(50 \mathrm{mM}$ Tris- $\mathrm{HCl}, \mathrm{pH} 8.5,1 \mathrm{mM}$ EDTA, $0.5 \%$ Tween- 20 and $200 \mu \mathrm{g} / \mathrm{ml}$ proteinase $\mathrm{K}$ ) at $55^{\circ} \mathrm{C}$. The enzyme was then inactivated by incubation at $95^{\circ} \mathrm{C}$ for 10 minutes. The control region of the mitochondrial genome was amplified as described previously 37 with the following modification: secondary PCR reactions were performed with 4 sets of overlapping M13-tailed primers (primer nucleotide positions: D1F: 15758-15777 and D1R: 019-001, D2F: 16223-16244 and D2R: 129-110, D3F: 16548-16569 and D3R: 389-370, D4F: 323-343 and D4R: 771-752) with an annealing temperature of $58^{\circ} \mathrm{C}$. PCR products were purified using TSAP (Promega) then sequenced on an ABI3130 Genetic Analyser (Applied Biosystems) with BigDye Terminator cycle sequencing chemistries (v3.1, Applied Biosystems). Sequences were directly compared to the revised Cambridge Reference Sequence for human mtDNA38 (GenBank Accession number: AC_000021.2) using SeqScape software (v2.1.1, Applied Biosystems). 


\section{Generation of heteroplasmic control DNA}

The mtDNA control region containing either the wild-type or polymorphic nucleotide of interest was amplified using PCR primers (primer nucleotide positions: forward primer 16016-16036, reverse primer 571-552) with an annealing temperature of $58^{\circ} \mathrm{C}$. PCR products amplified from ovarian follicular cells were purified using the Agencourt AMPure XP purification system (Beckman Coulter) according to the manufacturer's instructions. PCR products amplified from cumulus cells were gel purified (QIAquick Gel Extraction kit, Qiagen) and cloned using the pGEM®-T Easy Vector System (Promega) according to the manufacturer's instructions. Plasmid DNA was isolated using the QIAprep Spin Miniprep kit (Qiagen). Quantitative real-time PCR was performed using Platinum ${ }^{\circledR}$ SYBR $®$ Green qPCR SuperMix-UDG (Invitrogen) and PCR primers (forward primer L16016-16036, reverse primer, H16186-16167) to accurately determine the DNA concentration. Equimolar concentrations of DNA containing the wild-type or polymorphic nucleotide of interest were then combined in varying ratios to generate a range of heteroplasmic controls.

\section{Pyrosequencing}

Quantitative pyrosequencing was used to measure mtDNA carryover in samples from ePNT blastocysts and PNT-hESC lines. Locus-specific PCR and a pyrosequencing primer were designed for each polymorphic nucleotide of interest the mtDNA using PyroMark Assay Design Software v2.0 (Qiagen). Clumps of cells from ePNT blastocysts and from PNT$\mathrm{hESC}$ lines were lysed for 2 hours in a lysis buffer (50mM Tris-HCl, $\mathrm{pH} 8.5,1 \mathrm{mM}$ EDTA, $0.5 \%$ Tween- 20 and $200 \mu \mathrm{g} / \mathrm{ml}$ proteinase $\mathrm{K}$ ) at $55^{\circ} \mathrm{C}$. The enzyme was then inactivated by incubation at $95^{\circ} \mathrm{C}$ for 10 minutes. MtDNA amplification was performed prior to pyrosequencing analysis and pyrosequencing performed on the PyroMark Q24 and PyroMark Q96 instruments according to the manufacturer's instructions. Quantification of the heteroplasmy level was achieved using the instrument software to directly compare the relevant peak heights of both the wild-type and polymorphic nucleotides at the relevant position14. A standard curve was generated by plotting expected heteroplasmy level against actual heteroplasmy level for the control samples. The standard curve was used to determine the level of heteroplasmy in the blastocyst and PNT-hESC samples.

\section{Mitochondrial haplogroups}

Haplogroups were determined by next generation sequencing analysis of whole mtDNA, amplified in two overlapping 9kb fragments using primers L550-569 and H9839-9819 (set 1) and L9592-9611 and H645-626 (set 2), on an Ion Torrent Personal Genome Machine (Life Technologies) 39. Protocol modifications included use of a OneTouch 2 system and HiQ OT2 and sequencing kits. Samples were processed on Ion 316 chips and analysed with Torrent Suite Variant Caller plugin (version 4.6).

\section{Embryonic stem cell derivation}

For inner cell mass (ICM) isolation day 6 blastocysts were dissected in the embryo culture dish. The blastocysts were held in position by a holding pipette (Vitrolife, Sweden) with the ICM at 3 o'clock. The ICM was held in position with a biopsy pipette (Origio, Cat. No. MPB-FP-30) and isolated from the majority of the trophectoderm cells by laser pulses 
(Saturn 5 Active, Research Instruments). The isolated ICMs were plated on inactivated feeders MEF (CF1s), in medium containing KnockOut Serum Replacement (Invitrogen. Cat No. 10828), KnockOut DMEM (Invitrogen. Cat No. 10829), NEAA (Invitrogen. Cat No. 11140), bFGF2 (Invitrogen. Cat No. 13256), Glutamax (Invitrogen. Cat No. 35050) and 2Mercaptoethanol (Invitrogen. Cat No. 21985). Outgrowths were mechanically dissected from the surrounding cells and plated on fresh feeder cells, all subsequent passaging was performed mechanically. Embryonic stem cells were adapted to feeder-free culture conditions and maintained in mTeSR1 (STEMCELL Technologies) on growth factor reduced Matrigel (BD Biosciences).

\section{Quantitative RT-PCR (qRT-PCR)}

RNA was isolated using TRI Reagent (Sigma) and DNaseI treated (Ambion). cDNA was synthesized using a Maxima First Strand $c D N A$ Synthesis Kit (Fermentas). qRT-PCR was performed using Quantace Sensimix on an Applied Biosystems 7500 machine (Life Technologies Corporation, California, USA). Primer pairs used were previously published40 and are as follows: NANOG forward GCAACCTGAAGACGTGTGAA, reverse CTCGCTGATTAGGCTCCAAC; POU5F1 forward TATGGGAGCCCTCACTTCAC, reverse CAAAAACCCTGGCACAAACT; $S O X 2$ forward TTGTTCGATCCCAACTTTCC, reverse ACATGGATTCTCGGCAGACT.

\section{Immunohistochemistry and imaging}

Samples were fixed in $4 \%$ paraformaldehyde at $4{ }^{\circ} \mathrm{C}$ overnight, permeabilized with $0.5 \%$ Tween in 1X PBS for 20 minutes and blocked with 10\% FBS diluted in $0.1 \%$ Tween in $1 \mathrm{X}$ PBS for 1 hour. Primary antibodies were diluted in blocking solution as indicated: AFP (Dako, A0008, 1:500), Desmin (Neomarkers/Thermo, RB-9014-P, 1:50), Nanog (R\&D, AF1977, 1:500), Oct4 (Santa Cruz, SC-5279, 1:500), SMA (Sigma, A5228, 1:250), Sox 1 (R\&D, AF3369, 1:500), Sox2 (Cell Signalling, 23064, 1:500), Sox17 (R\&D, AF1924, 1:500), SSEA4 (DSHB, MC-813-70, 1:100), Tuj1 (Sigma, T2200, 1:500). Samples were incubated at $4{ }^{\circ} \mathrm{C}$ rotating overnight. Alexa Fluor secondary antibodies (Invitrogen, antimouse A21202, A21203; anti-rabbit A21206, A21207; anti-goat A11055, A11058) were diluted 1:300 in blocking solution and samples incubated for 1 hour at room temperature, then washed and covered with $0.1 \%$ Tween in $1 \mathrm{X}$ PBS containing DAPI Vectashield mounting medium (Vector Labs). Images were taken on an Olympus 1 X73 microscope with Cell^F software (Olympus Corporation, Tokyo, Japan).

\section{Statistical Analysis}

Data were analysed using one-way ANOVA with Tukey's HSD test, Mann-Whitney U test, $\chi^{2}$ test and Fisher's exact test, as indicated in the figure legends. RNA-seq data were analysed by PCA using either RPKM- or VST-normalized counts. 


\section{Extended Data}

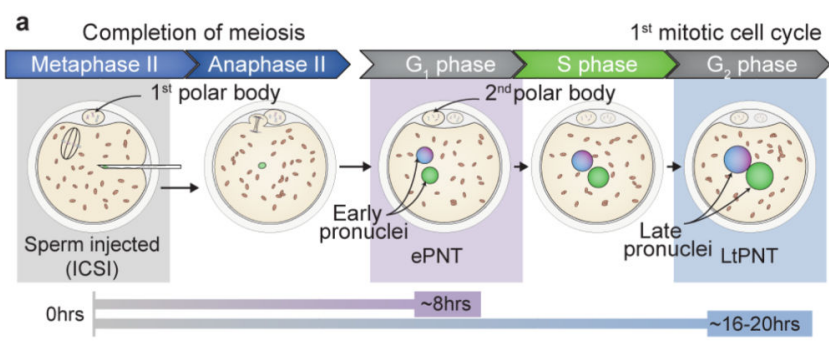

c

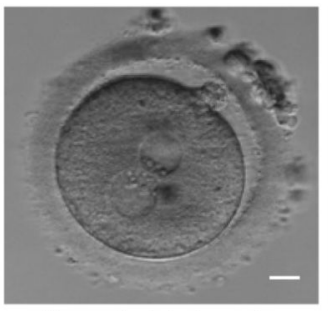

Unmanipulated zygote

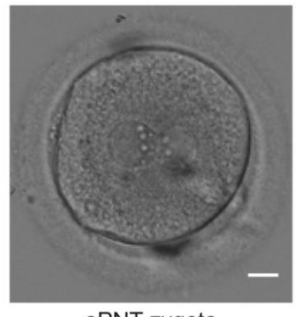

ePNT zygote b LtPNT

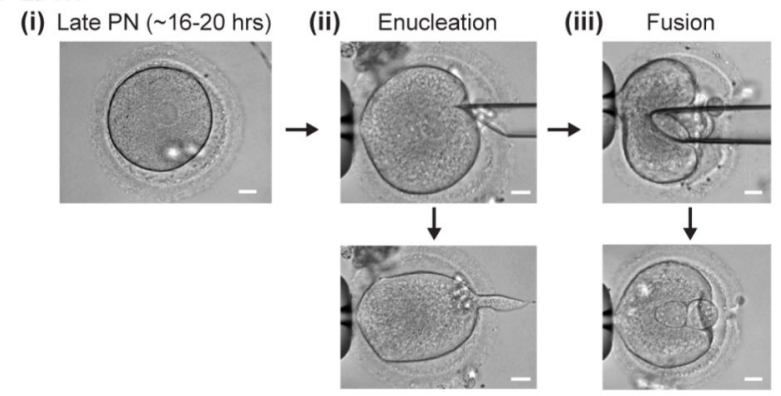

d

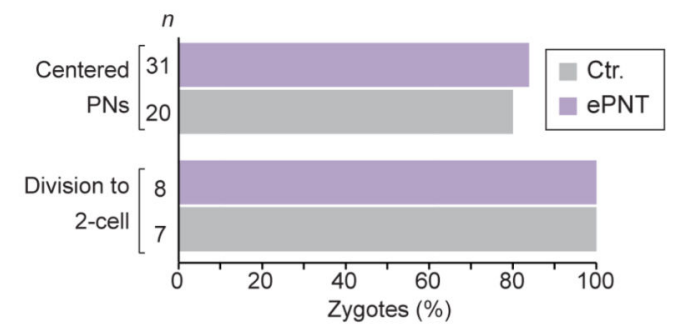

Extended Data Figure 1. Late PNT and PN centralisation following early PNT.

a, Schematic showing timing of late PNT (LtPNT) and early PNT (ePNT). b, Images showing the stages of the LtPNT process.(i) late PN zygote (ii) enucleation (iii) fusion. Scale bar, $20 \mu \mathrm{m}$. Note large pronuclei and pipette size compared with Fig. $1 \mathrm{~b}$ and Videos 1 and 2. c, Images show unmanipulated and ePNT zygotes at $16-18 \mathrm{hr}$ after fertilization. Scale bars, $20 \mu \mathrm{m}$. d, PN centralization and division to the 2-cell stage assessed by live cell imaging in control and ePNT zygotes (not significant). Comparisons by $\chi^{2}$ test. 
a

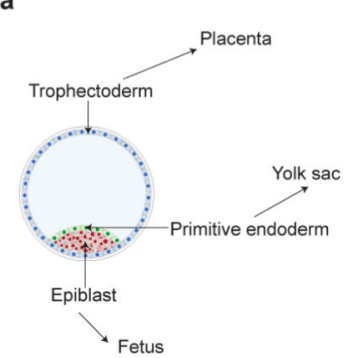

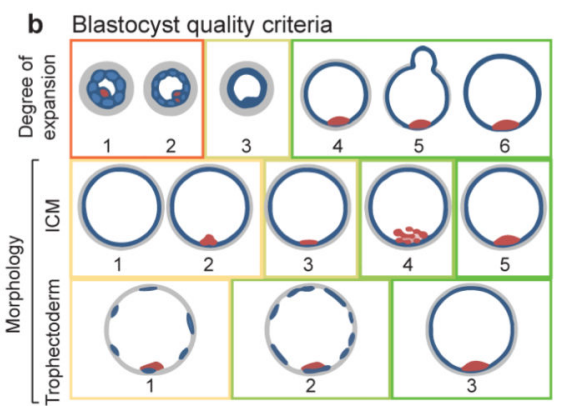

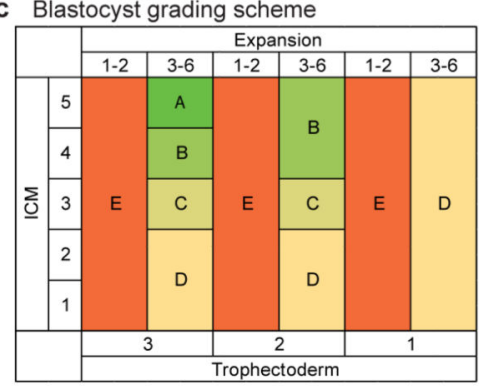

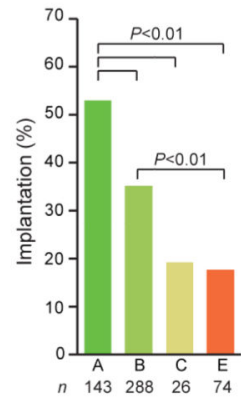

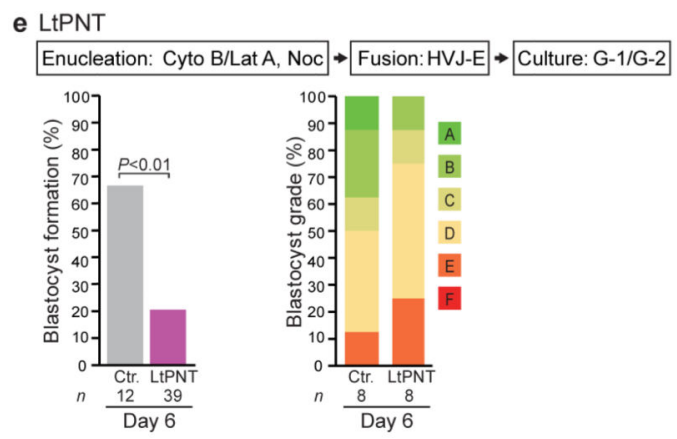

f ePNT (series I)

Enucleation: Sucrose, Lat A, Noc $\rightarrow$ Fusion: HVJ-E $\rightarrow$ Culture: G-1/G-2
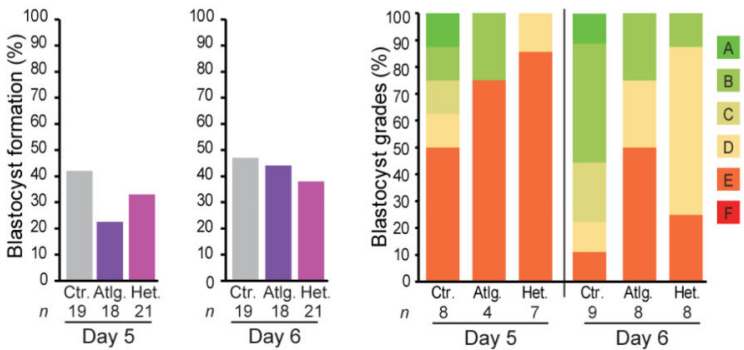

g

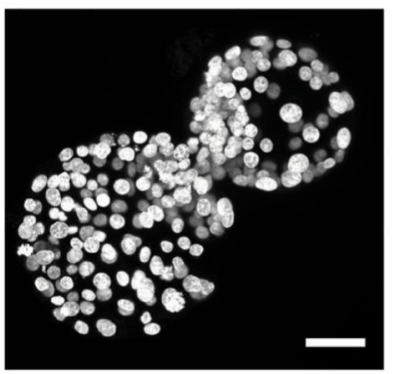

h

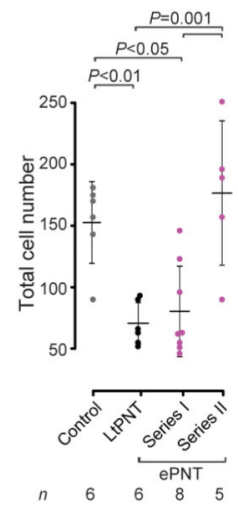

Extended Data Figure 2. Blastocyst morphology and effect of PNT on blastocyst development and quality.

a, Schematic showing cell lineages in a mammalian blastocyst: Trophectoderm (TE); Primitive endoderm (PE) and Epiblast (Epi). b, Morphological criteria and scoring system used for grading human blastocysts 31 . Top panel shows degree of expansion ranging from an early, unexpanded blastocyst (score 1) to fully expanded (score 6). Middle panel shows a range of inner cell mass (ICM) morphologies ranging from absent (score 1) to large but tightly packed (score 5). Bottom panel shown a range of trophectoderm (TE) morphologies ranging from scant and discontinuous (score 1) to a fully formed layer of tightly packed cells (score 3). Box colours correspond to the grades shown in c. c, Table used to assign blastocyst grades, according to levels of expansion, and morphology of the ICM and TE. 
Grade F (not shown) was assigned to embryos that developed to the blastocyst stage but subsequently showed signs of degeneration. d, Graph showing the relationship between blastocyst grade and implantation. Data obtained from clinical IVF cycles $(n=531)$ in which unmanipulated single blastocysts were replaced on day 5. Implantation is defined by the detection of a fetal heartbeat at 6 weeks after IVF treatment. There was no case in which a grade D or F blastocyst was replaced. $P$ values are shown ( $\chi^{2}$ test). e, LtPNT experimental conditions, blastocyst formation $\left(P<0.01 ; \chi^{2}\right.$ test) and quality. A total of 51 zygotes from 10 donors were allocated either to an unmanipulated control group (Ctr, $n=12)$ or to LtPNT involving transfer between pairs of zygotes from the same donor $(n=29)$ or replacement back into the same zygote (Autologous PNT (Atlg) $n=10$ ). f, ePNT (series I) experimental conditions, blastocyst formation and quality. This series of experiments involved a total of 58 zygotes from 13 donors. Zygotes were allocated to a control group ( $\mathrm{Ctr}, n=19)$, or to ePNT involving either autologous (Atlg, $n=18$ ) or heterologous (Het, $n=21$ ) transfers. Differences are not significant ( $\chi^{2}$ test and Fisher's exact test). g, Image of an ePNT

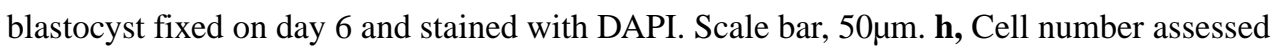
by nuclear counts showing comparable numbers in control and ePNT (Series II) blastocysts and significantly reduced numbers in LtPNT and ePNT (Series I) blastocysts ( $P=0.001$; oneway ANOVA with Tukey's HSD test). Mean \pm s.d. calculated from individual blastocysts, numbers indicated on the $\mathrm{x}$-axis. 

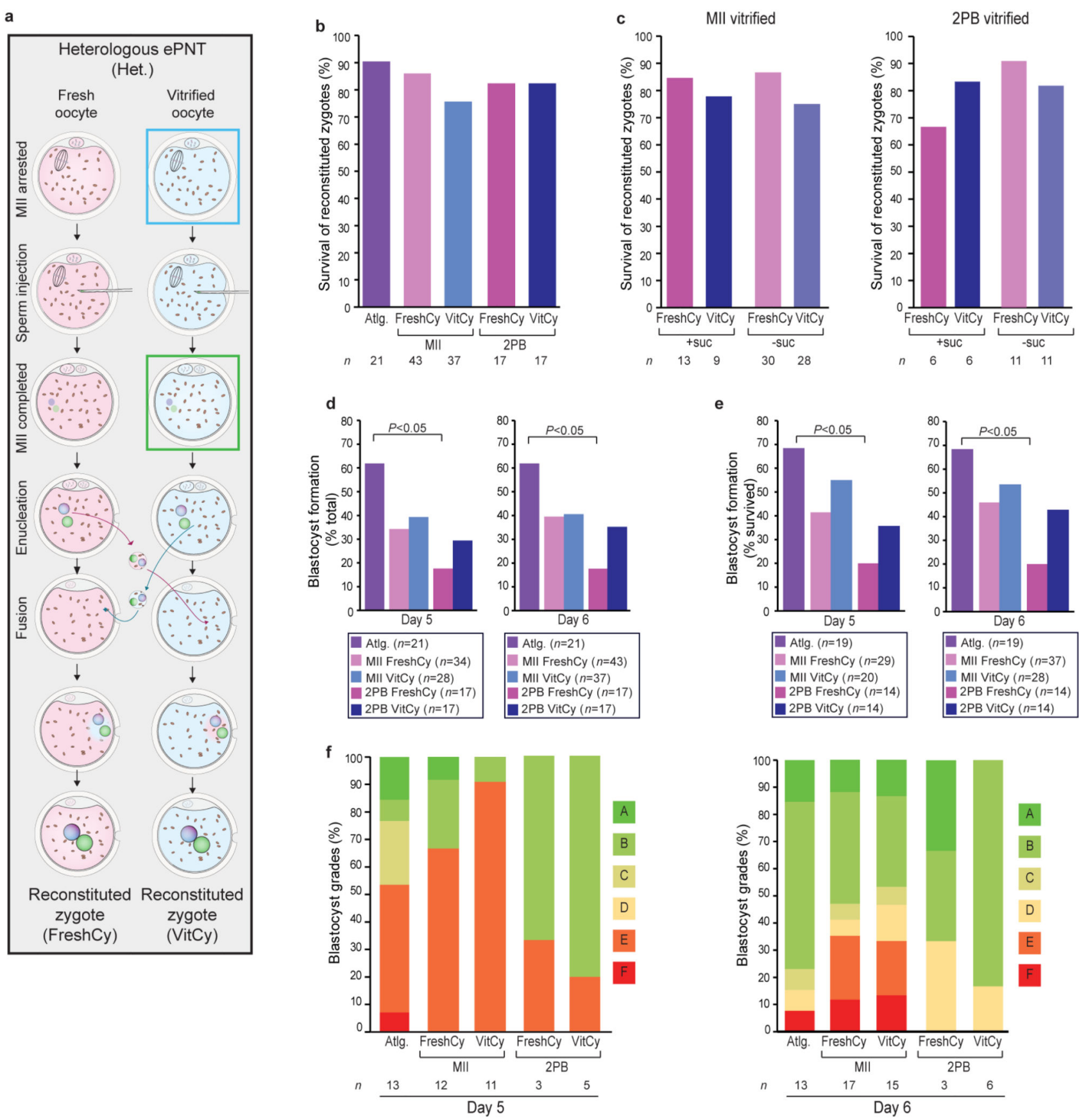

Extended Data Figure 3. Survival and blastocyst development following ePNT between zygotes obtained from freshly harvested and vitrified oocytes.

a, Experimental scheme for heterologous ePNT in Series II. Because of unpredictability in the response to ovarian stimulation, heterologous transfers involved reciprocal ePNT between zygotes generated from freshly harvested and vitrified oocytes. This resulted in reconstituted zygotes whose cytoplasts originated from a fresh oocyte (FreshCy), or from a vitrified oocyte (VitCy). Oocytes for these experiments were vitrified predominantly at the MII stage (blue box; $n=80$ zygotes; 25 donors). We also conducted a series of experiments to 
determine whether vitrification at the $2 \mathrm{~PB}$ stage (green box; $n=34$ zygotes; 13 donors) would promote improved blastocyst formation. $\mathbf{b}$, Survival of reconstituted zygotes as a proportion of those submitted to autologous (Atlg) and heterologous (Het) ePNT according to the stage of vitrification (MII or 2PB) and according to whether the cytoplast was derived from a fresh (FreshCy) or a vitrified (VitCy) oocyte. Loss was generally due to karyoplast lysis, excessive leakage of cytoplasm, or degeneration of reconstituted zygotes during subsequent incubation. Differences are not significant ( $\chi^{2}$ test). c, Sucrose was initially included in the manipulation medium to facilitate enucleation and fusion, however it was later removed because the data indicated that the osmotic effect resulted in increased mtDNA carryover (see Fig. 4b). Omission of sucrose from the enucleation medium had a small, but not significant, effect on survival of zygotes whose cytoplasts originated from vitrified MII oocytes ( $\chi^{2}$ test). d, Blastocyst formation as a percentage of zygotes submitted to the ePNT procedure recorded on days 5 and 6 following fertilisation. e, Blastocyst formation recorded on days 5 and 6 as a percentage of zygotes that survived the ePNT procedure. The numbers in each group and $P$ values are shown, $\chi^{2}$ test. f, Blastocyst quality grades (see Extended Data Fig. 2 c,d) on days 5 and 6 (not significant; Fisher's exact test). (Source data files available online). 


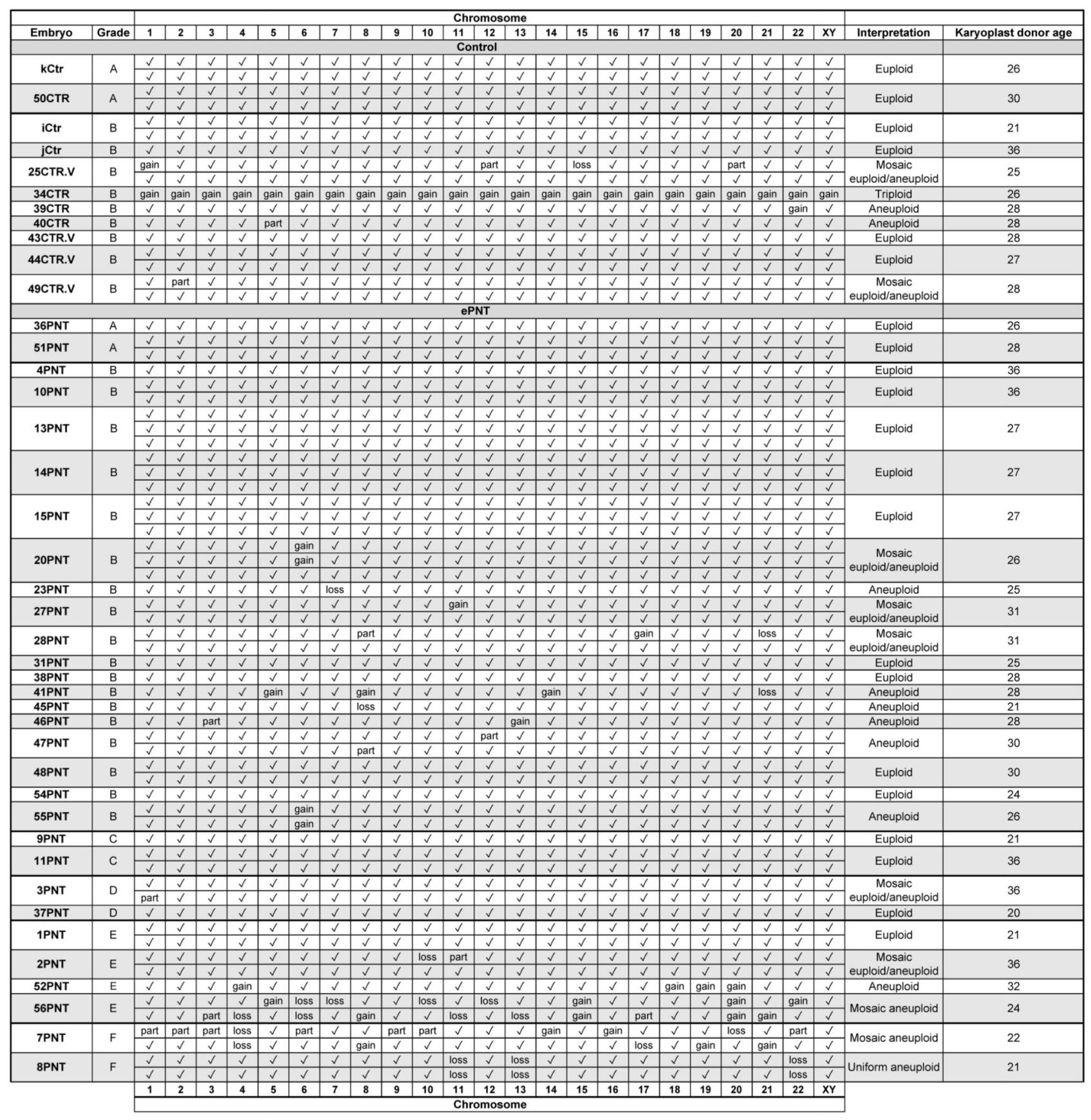

Extended Data Figure 4. Array-CGH results for PNT blastocysts.

Summary of array-CGH results obtained from inner cell mass and trophectoderm samples from control $(n=11)$ and ePNT ( $n=30)$ blastocysts. Blastocysts are ordered by grade within experimental groups. The karyoplast donor age is also shown. 

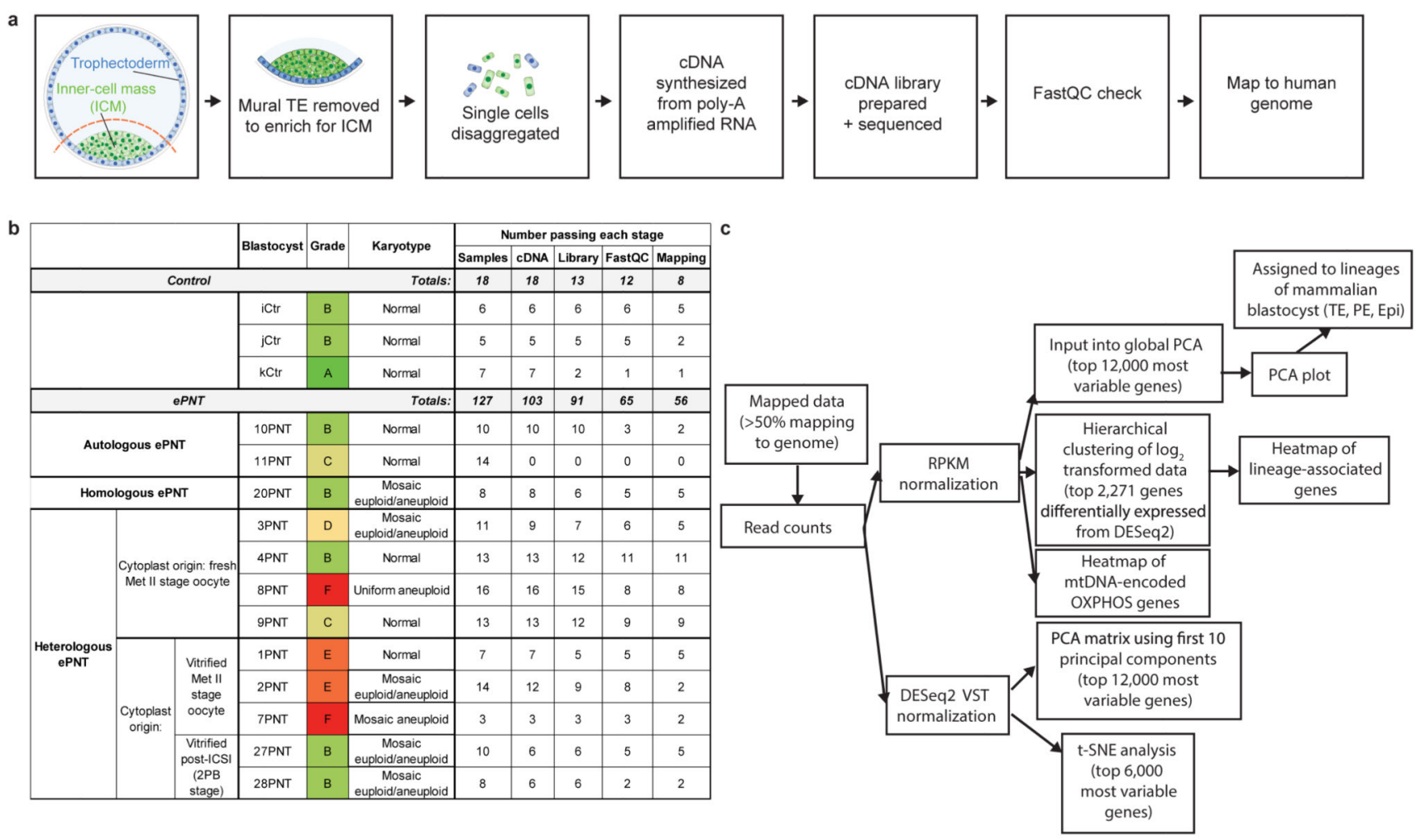

Extended Data Figure 5. Experimental approach and bioinformatics analysis of single cell RNAseq data from ePNT and control blastocysts.

a, Flow diagram showing the steps involved in RNA-seq of single cells microdissected from human blastocysts. b, Summary table of control and ePNT blastocysts submitted for RNAseq analysis. For the purpose of gene expression analysis, we distinguish between ePNT blastocysts derived from fusion of cytoplasts and karyoplasts with the same and different mitochondrial genomes. Those with the same mitochondrial genomes included blastocysts from autologous ePNT and from zygote pairs donated by two sisters, which we refer to as homologous ePNT. Blastocysts arising from heterologous ePNT represent new combinations of nuclear and mitochondrial genome and are sub-grouped according to the cytoplast origin (see Extended Data Fig. 4). c, Flow diagram outlining the bioinformatics analysis of RNAseq data. Data were normalized either as reads per kilobase per million mapped reads (RPKM)41 or using DESeq236 Normalized data were used to generate principal component analysis (PCA) plots, t-SNE plots and heatmaps. RNA-seq data has been deposited to Gene Expression Omnibus (GSE76284). 


\begin{tabular}{|c|c|c|c|}
\hline & Cells & Blastocysts & Grade \\
\hline Ref. + Ctr. & 38 & 11 & A/B \\
\hline ePNT $\left[\begin{array}{l}\text { Atlg./Homol. } \\
\text { Het. }\end{array}\right.$ & 7 & 2 & B \\
& 27 & 4 & A-C \\
\hline
\end{tabular}

b

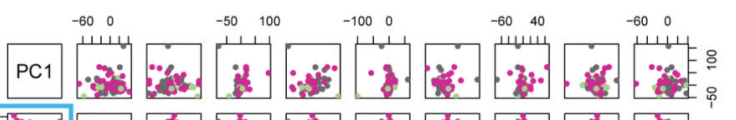

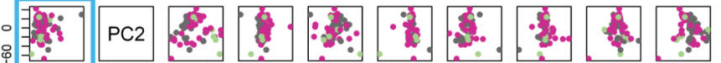

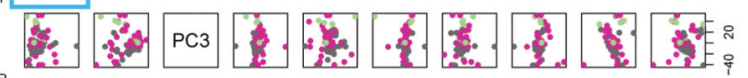

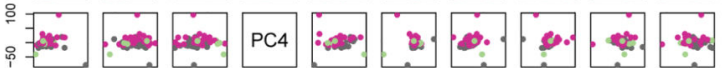

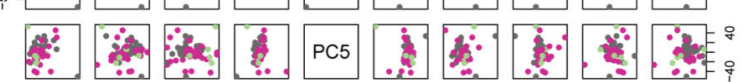

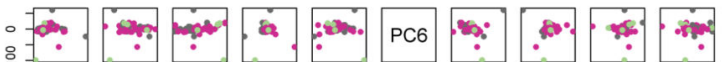

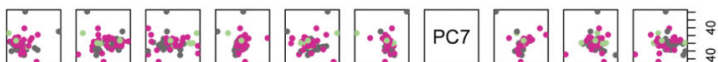

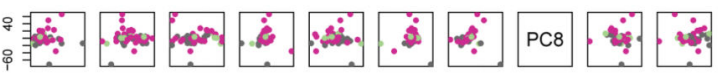
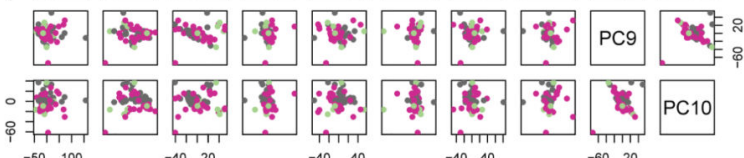

$\begin{array}{lll}-50 & 100\end{array}$

$-40 \quad 20$

$-40 \quad 40$

$-40 \quad 40$

$-6020$

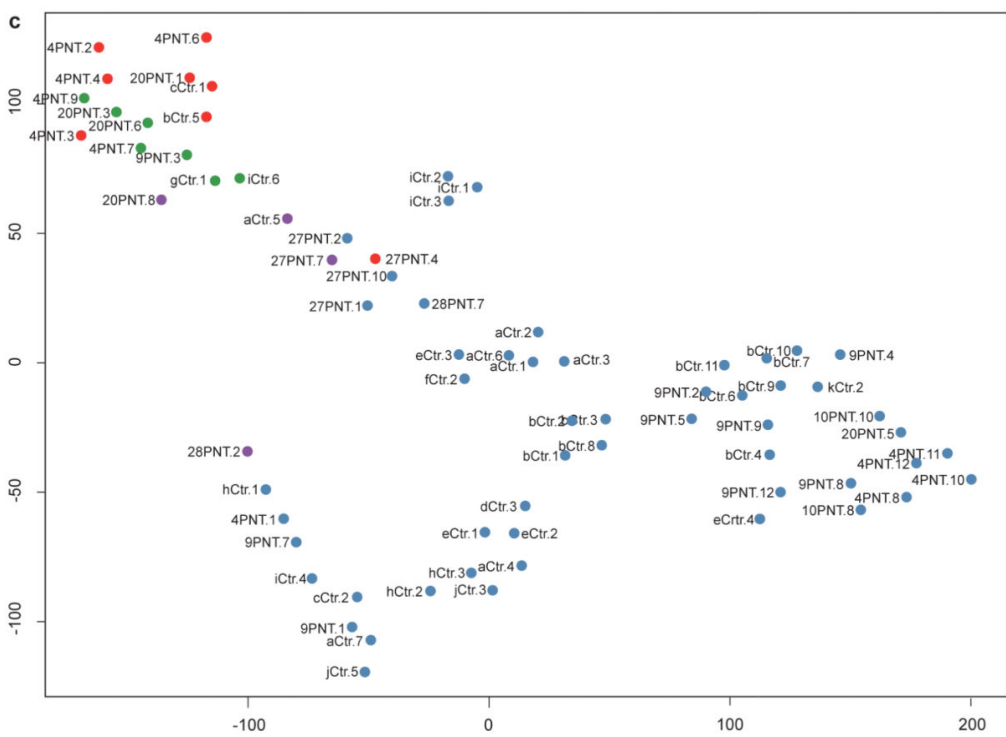

Primitive endoderm ( $n=8$ samples; 5 blastocysts) Epiblast ( $n=7$ samples; 5 blastocysts) - Trophectoderm ( $n=53$ samples; 16 blastocysts) Ambiguous ( $n=4$ samples; 4 blastocysts)

Extended Data Figure 6. Analysis of differential gene expression in good quality ePNT and control blastocysts.

a, PCA matrix using the first ten principal components of DESeq2 VST normalized data for the top 12,000 most variable genes. Global gene expression is indistinguishable between unmanipulated control and ePNT samples, PC1 versus PC2 highlighted in blue box. b, PCA matrix as shown in a., distinguished by lineage, clearly seen in PC2 versus PC3 (pink box). c, t-SNE analysis following DESeq2 VST normalization of 6,000 of the most variably expressed genes, where samples were distinguished by lineage. Sample numbers and 
blastocyst grades are shown. Autologous and homologous ePNT samples are derived from blastocysts in which the karyoplast and cytoplast had the same mitochondrial genome.

Heterologous ePNT samples were derived from pairs of zygotes with different mitochondrial genomes (Extended Data Fig. 5). Samples from experimental controls and reference population were combined for the purpose of the analyses shown in a and $b$.
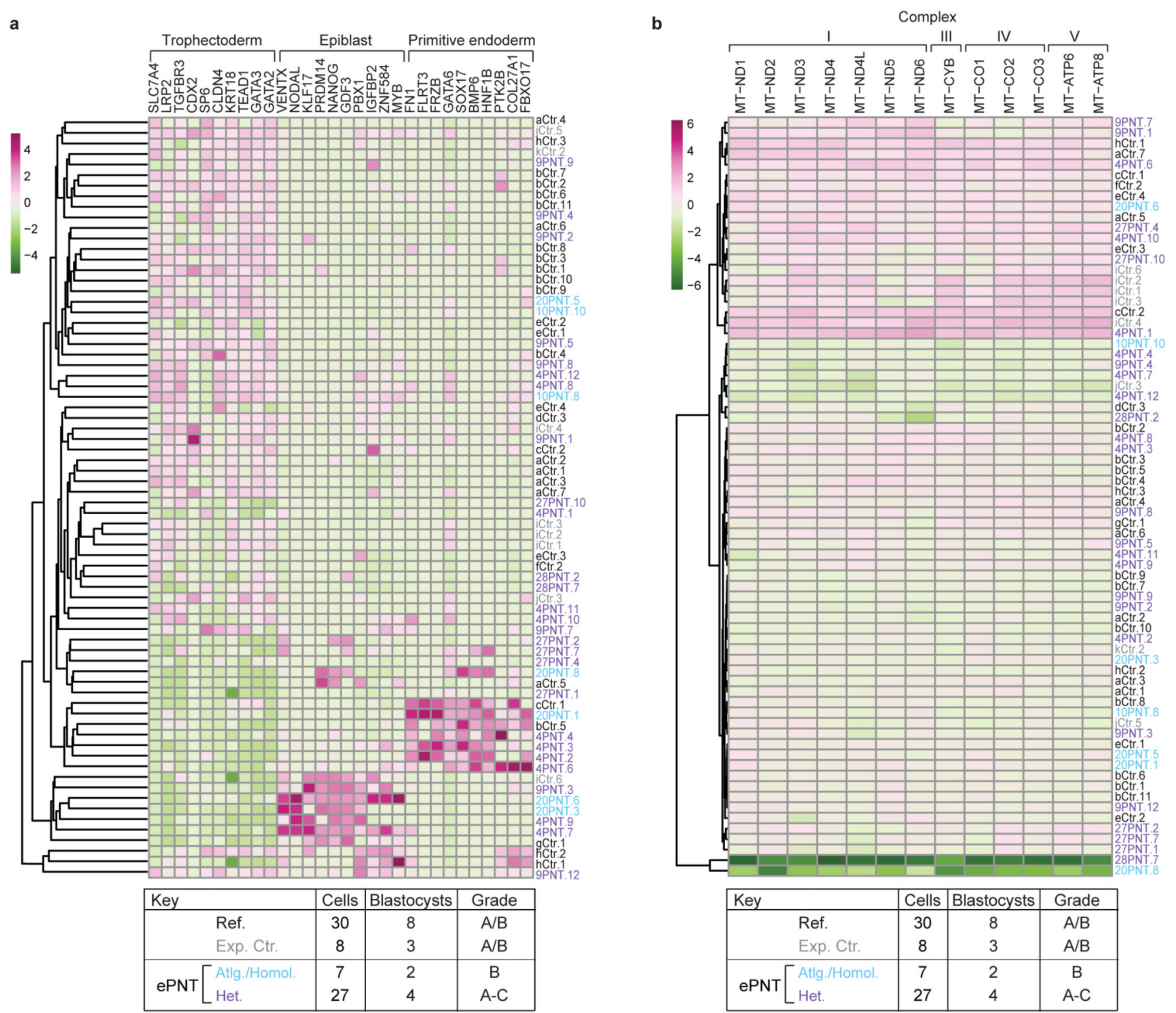

Extended Data Figure 7. Expression of lineage specific genes and mitochondrial OXPHOS genes in control and ePNT embryos.

a, Heatmap showing $\log _{2}$ transformed RPKM values of selected differentially expressed genes in trophectoderm $(n=10)$, epiblast $(n=10)$ and primitive endoderm $(n=10)$ lineages. $\mathbf{b}$, Heatmap showing expression of mitochondrial OXPHOS genes following unsupervised hierarchical clustering. Expression of OXPHOS genes encoded by mtDNA is variable both within and between blastocysts. Control and ePNT samples cluster together, irrespective of whether the cytoplast and karyoplast had the same (blue font) or different (purple font) 
mtDNA. Sample numbers and blastocyst grades are shown. The reference population includes a previously published series14. Autologous and homologous ePNT samples are derived from blastocysts in which the karyoplast and cytoplast had the same mitochondrial genome. Heterologous ePNT samples were derived from pairs of zygotes with different mitochondrial genomes (Extended Data Fig. 5b). Expression levels are indicated on a highto-low scale (purple-white-green). (Source data files available on line for parts a and $b$ )

a

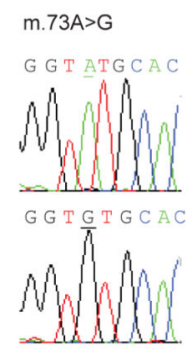

b
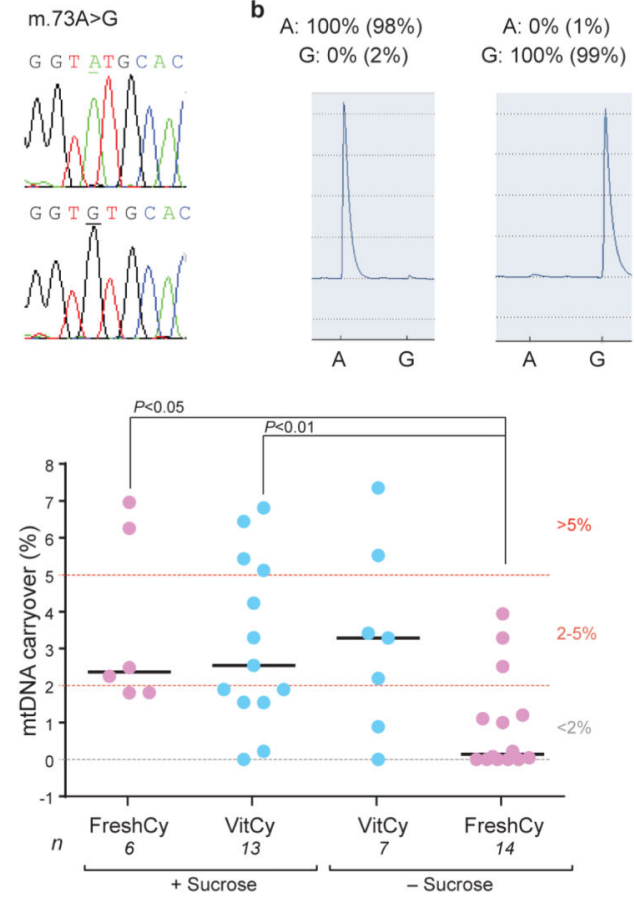
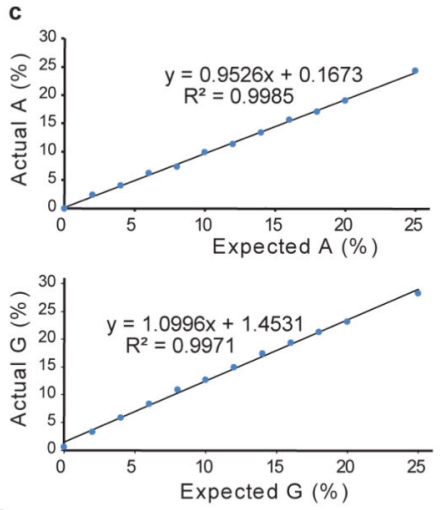

$\mathbf{f}$

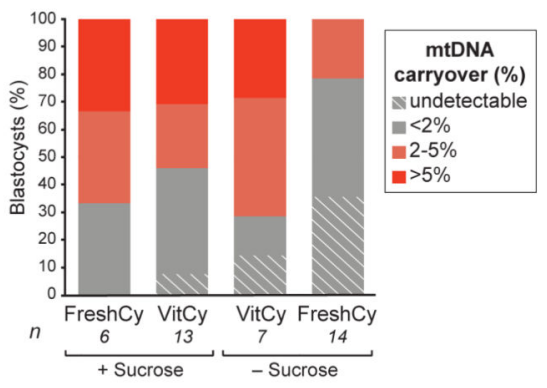

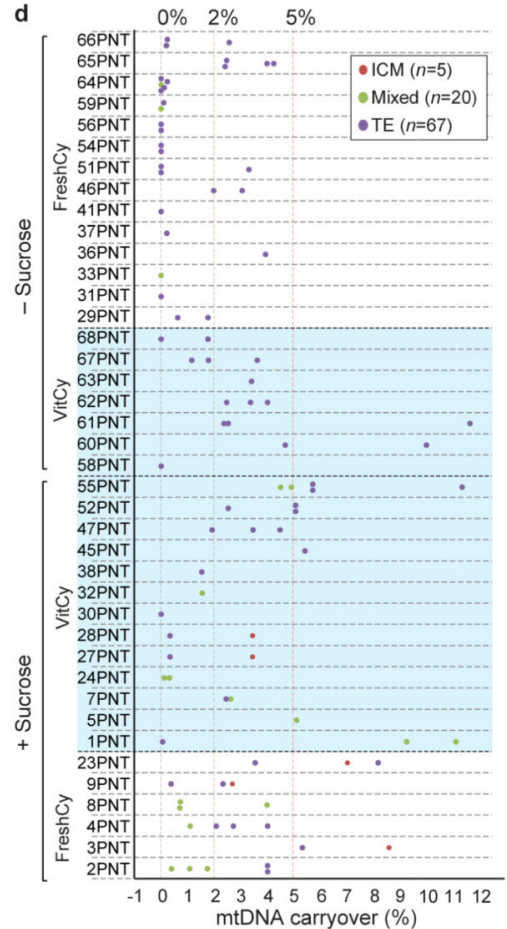

Extended Data Figure 8. Measurement of heteroplasmy due to mtDNA carryover during ePNT. a, Mitochondrial genotypes were determined by identifying polymorphic variants in the hypervariable mtDNA control regions of each donor. Sequence electropherograms of mtDNA non-coding control region with a sequence variant used for pyrosequencing (highlighted) (m.73A>G). b, Sequence pyrograms for the mtDNA variant $(m .73 A>G)$ in control samples. The expected level of variant is given along with the level determined by pyrosequencing (in brackets). c, Examples of the standard curve generated to increase accuracy in detecting low levels (0-25\%) of heteroplasmy by pyrosequencing, which has previously been reported to accurately detect heteroplasmy at a level of $1 \% 42$. Each data point represents the mean of 3-4 technical replicates. d, MtDNA carryover was measured by pyrosequencing using clumps of cells $(n=92)$ from day 6 blastocysts ( $n=40$; names shown on $\mathrm{y}$ axis). The cells were predominantly obtained from the TE layer (purple, $n=67$ ). ICM cells (red, $n=5$ ) and cells of mixed TE/ICM origin (green, $n=20$ ) were also analysed. Each data point represents the mean of 2-3 technical replicates. e, MtDNA carryover from individual blastocysts calculated from data shown in d. Each data point represents either the mean value where $>1$ sample was tested ( $n=28$ ePNT blastocysts), or a single value where only one sample was tested ( $n=12$ ePNT blastocysts). Horizontal lines show median values for each experimental group. Blastocysts arising from ePNT performed in the absence of 
sucrose and fused with a fresh cytoplast (FreshCy) had significantly reduced mtDNA carryover compared with blastocysts where ePNT was performed in the presence of sucrose ( $P$ values and blastocyst numbers are shown; two-sided Mann-Whitney U test). f, Graph showing the proportions of blastocysts $(n=40)$ with mtDNA carryover measurements falling within the specified levels (not significant: $\chi^{2}$ test). (Source data files provided for parts cf).
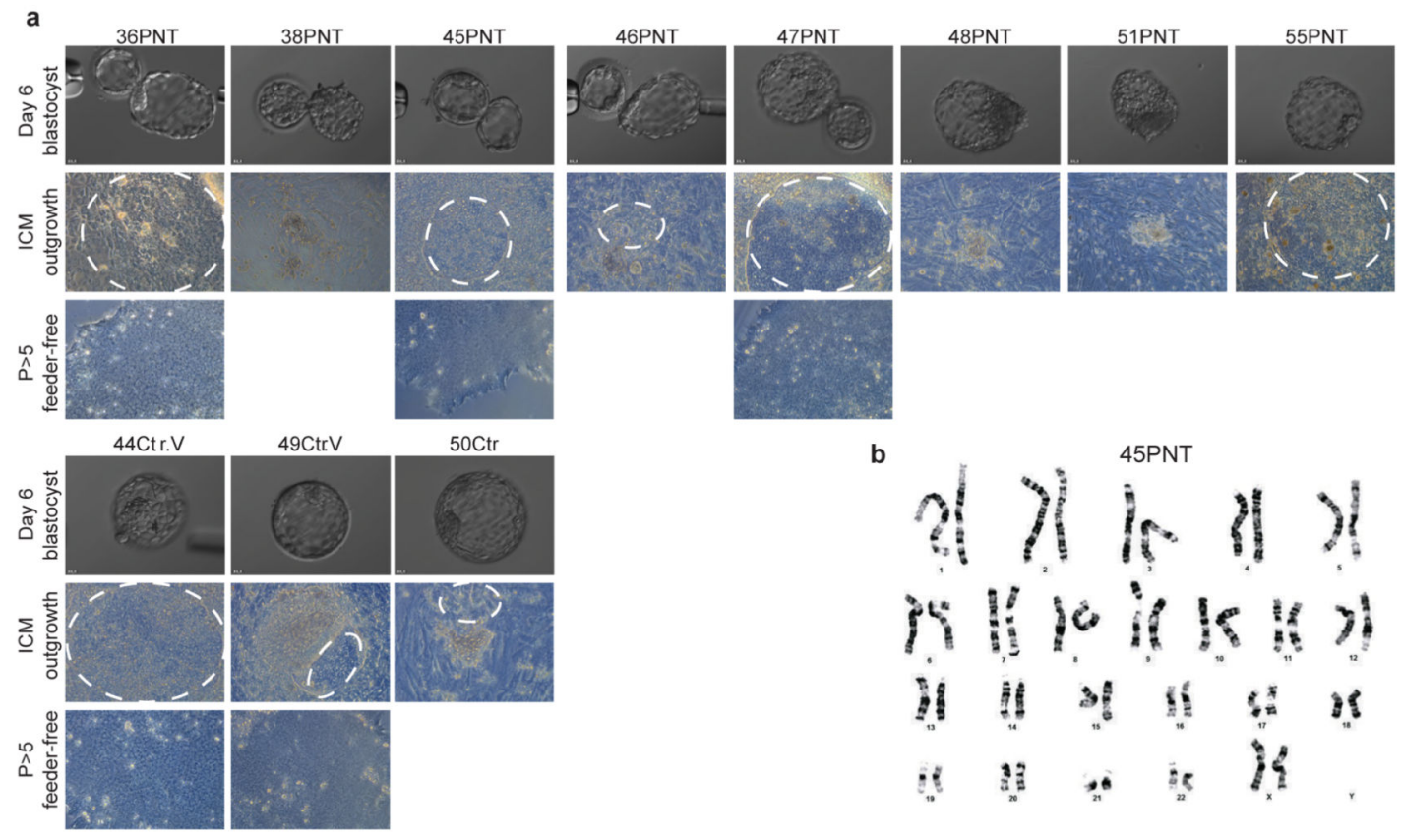

C
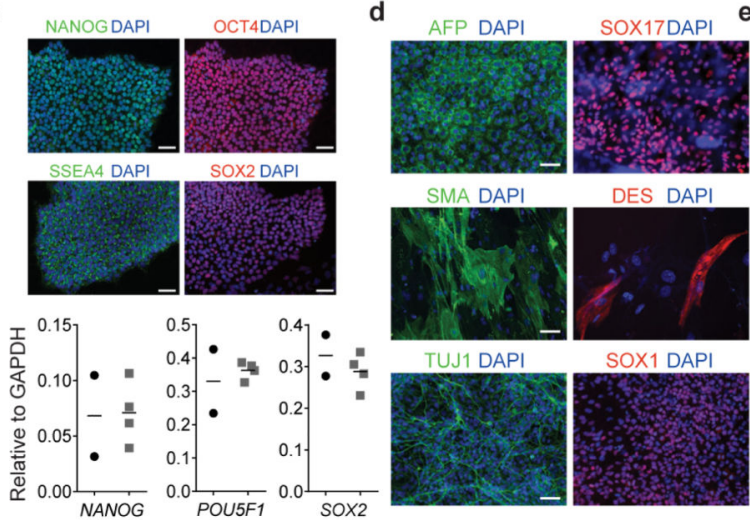

e

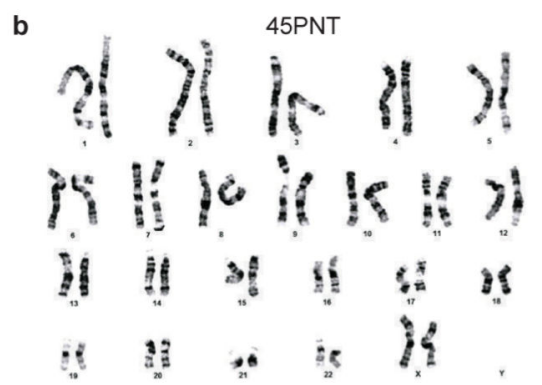

- Ctr. $(n=2)$ ePNT $(n=4)$

\begin{tabular}{|c|c|c|c|}
\hline ES cell line & $\begin{array}{l}\text { mtDNA } \\
\text { sequence } \\
\text { variant }\end{array}$ & $\begin{array}{l}\text { PCR amplification } \\
\text { (Forward/Reverse primers) }\end{array}$ & Pyrosequencing Primer \\
\hline 31PNT & $\mathrm{m} .499 \mathrm{G}>\mathrm{A}$ & $\begin{array}{l}\text { 5' ACATTATITTCCCCTCCCACTCC } 3^{\prime} \\
\text { Bio-5' GTTCGGGGTATGGGGTTAGCA 3' }\end{array}$ & 5' CTAATCTCATCAATACAACC 3' \\
\hline 36PNT & $\mathrm{m} .73 \mathrm{~A}>\mathrm{G}$ & $\begin{array}{c}5^{\prime} \text { TAACCACTCACGGGAGCTCT } 3^{\prime} \\
\text { Bio-5' AGGCAGGAATCAAAGACAGATACT } \\
3^{\prime}\end{array}$ & 5' TाTCGTCTGGGGGGT 3' \\
\hline 45PNT & $\mathrm{m} .73 \mathrm{~A}>\mathrm{G}$ & $\begin{array}{c}5^{\prime} \text { TAACCACTCACGGGAGCTCT } 3^{\prime} \\
\text { Bio-5' AGGCAGGAATCAAAGACAGATACT } \\
3^{\prime}\end{array}$ & 5' TाTCGTCTGGGGGGT 3' \\
\hline 47PNT & m.16126T>C & $\begin{array}{l}\text { 5' CCCATCAACAACCGCTATGTATाT 3' } \\
\text { Bio-5' AGGGGGTITGATGTGGATTG 3' }\end{array}$ & 5' AGCCACCATGAATATTG 3' \\
\hline 55PNT & $\mathrm{m} .73 \mathrm{~A}>\mathrm{G}$ & $\begin{array}{c}\text { 5' 'AACCACTCACGGGAGCTCT 3' } \\
\text { Bio-5' AGGCAGGAATCAAAGACAGATACT } \\
3^{\prime}\end{array}$ & 5' TाTCGTCTGGGGGGT 3' \\
\hline
\end{tabular}

\begin{tabular}{|c|c|c|c|c|c|c|c|c|}
\hline & & & \multicolumn{2}{|c|}{ Aneuploidy } & \multicolumn{2}{c|}{ Haplogroup (donor) } & \multicolumn{2}{c|}{ mtDNA carryover (\%) } \\
\hline Embryo & Grade & Origin & Blastocyst (TE) & hESC & Karyoplast & Cytoplast & Blastocyst (TE) & hESC \\
\hline 31PNT & B & Fresh Cy & Normal (1/1) & Normal (2/2) & K & U & Undetectable & Undetectable \\
\hline 36PNT & A & Fresh Cy & Normal (1/1) & Normal (13/15) & $\mathrm{H}$ & $\mathrm{H}$ & 3.94 & $0-51.99$ \\
\hline 45PNT & B & VitCy & Aneuploid (1/1) & Normal (6/7) & L0 & $\mathrm{H}$ & 5.29 & $0-4.43$ \\
\hline 47PNT & B & VitCy & Aneuploid (2/2) & Normal (11/12) & $\mathrm{J}$ & $\mathrm{U}$ & $3.55 \pm 1.41$ & $0-8.21$ \\
\hline 55PNT & B & Vit Cy & Aneuploid (2/2) & Not tested & $\mathrm{H}$ & $\mathrm{K}$ & $6.44 \pm 2.8$ & Undetectable \\
\hline
\end{tabular}

*Did not grow beyond passage 2

Extended Data Figure 9. Derivation and characterisation of human embryonic stem cells from control and ePNT blastocysts. 
a, Examples of outgrowths formed following explantation of the ICM from ePNT ( $n=15)$ and control $(n=6)$ blastocysts used for hESC derivations. The dashed white circle indicates the region picked for initial passage of the ICM outgrowth. Bottom panel shows examples of hESC colonies. b, Example of a normal karyogram from an PNT-hESC line (45PNT); 4/4 lines tested showed a normal karyotype. The remaining hESC line did not grow beyond passage 2 and was derived from a uniformly aneuploid blastocyst (55PNT; Extended Data Fig. 4). c, Immunostaining of representative PNT-hESCs (grown in mTeSR1) for NANOG, SSEA4 (green), SOX2 and OCT4 (red) with DAPI (blue) merge. Graph shows qRT-PCR analysis of control and PNT-hESC lines for pluripotency transcripts NANOG, POU5F1 and $S O X 2$. Horizontal line shows the median value, which is similar between hESCs from unmanipulated control blastocysts (Ctr; $n=2 \mathrm{hESC}$ lines) and ePNT-hESCs. ( $n=4$ ePNThESC lines). d, Immunostaining of representative PNT-hESCs after 20 days in basal MEF media, confirming differentiation into all three germ layers: endoderm (alpha-fetoprotein, AFP; SOX17), mesoderm (alpha-smooth muscle actin, SMA; desmin, DES) and ectoderm (beta-III tubulin, TUJ1; SOX1) in green or red, with DAPI (blue) merge. Scale bars, 50 $\mu$ m. e, Table showing the mtDNA variants and primers used to measure mtDNA carryover in PNT-hESC lines. f, Summary table showing details of blastocysts and the corresponding hESCs. Aneuploidy in PNT-hESC lines was analysed by metaphase spreads, except for 31PNT-hESC, which was determined by array-CGH. 

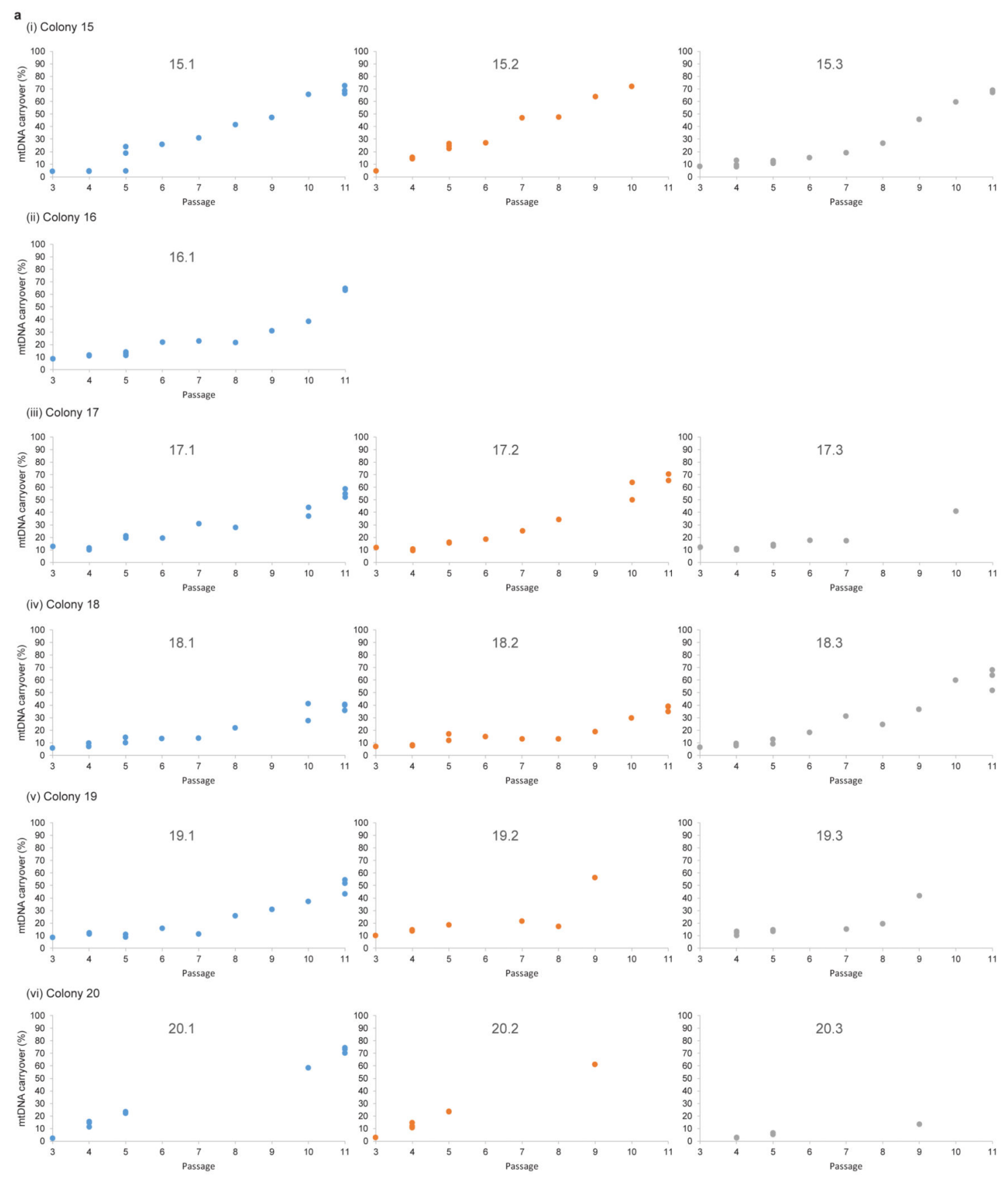

Extended Data Figure 10. Heteroplasmy in subclones of the hESC line derived from 36PNT a, The 36PNT hESCs line was frozen at passage 3 (after derivation), thawed and subcloned to monitor heteroplasmy arising from the karyoplast donor mtDNA. Six colonies (15-20) were randomly selected at the first post-thaw passage (P3) and clumps of cells were plated on 3 tissue culture wells; 5/6 colonies gave rise to 3 subclones which were grown to P11. Sub-clones are distinguished by colour in the graphs. Each data point represents the mean of two technical replicates for a single cell sample. (Source data file available online). 


\section{Supplementary Material}

Refer to Web version on PubMed Central for supplementary material.

\section{Acknowledgements}

We are very grateful to those who donated gametes for this research and we thank Maria Nesbitt and Kayleigh Lennox for obtaining their consent. We also thank Patrick Chinnery and Vasileios Floros for helpful discussion. The work was funded by the Wellcome Trust (096919) and by grants from the NIHR Newcastle Biomedical Research Centre and the Barbour Foundation. KKN and co-workers are supported by The Francis Crick Institute, which receives its core funding from Cancer Research UK, the UK Medical Research Council (MC_UP_1202/9) and the Wellcome Trust, and by the March of Dimes Foundation (FY11-436). DW and co-workers are funded by NIHR Oxford Biomedical Research Centre.

\section{References}

1. Schon EA, DiMauro S, Hirano M. Human mitochondrial DNA: roles of inherited and somatic mutations. Nat Rev Genet. 2013; 13:878-890.

2. Craven L, et al. Pronuclear transfer in human embryos to prevent transmission of mitochondrial DNA disease. Nature. 2010; 465:82-85. [PubMed: 20393463]

3. Wallace DC, Chalkia D. Mitochondrial DNA Genetics and the Heteroplasmy Conundrum in Evolution and Disease. Cold Spring Harbor Perspectives in Biology. 2013; 5

4. McFarland R, et al. Multiple neonatal deaths due to a homoplasmic mitochondrial DNA mutation. Nat Genet. 2002; 30:145-146. [PubMed: 11799391]

5. Steffann J, et al. Data from Artificial Models of Mitochondrial DNA Disorders Are Not Always Applicable to Humans. Cell Reports. 2014; 7:933-934. [PubMed: 24856293]

6. Richardson J, et al. Assisted reproductive technologies to prevent transmission of mitochondrial DNA disease. Stem Cells. 2015; 33:639-645. [PubMed: 25377180]

7. Tachibana M, et al. Towards germline gene therapy of inherited mitochondrial diseases. Nature. 2013; 493:627-631. [PubMed: 23103867]

8. Paull D, et al. Nuclear genome transfer in human oocytes eliminates mitochondrial DNA variants. Nature. 2013; 493:632-637. [PubMed: 23254936]

9. McGrath J, Solter D. Nuclear transplantation in the mouse embryo by microsurgery and cell fusion. Science. 1983; 220:1300-1302. [PubMed: 6857250]

10. Fenwick J, Murdoch AP, Herbert M. Time from insemination to first cleavage predicts developmental competence of human pre-implantation embryos. Human Reproduction. 2002; 17:407-412. [PubMed: 11821286]

11. Schatten H, Sun QY. The role of centrosomes in mammalian fertilization and its significance for ICSI. Mol Hum Reprod. 2009; 15:531-538. [PubMed: 19549764]

12. Niakan KK, Han J, Pedersen RA, Simon C, Pera RAR. Human pre-implantation embryo development. Development. 2012; 139:829-841. [PubMed: 22318624]

13. Forman EJ, et al. Oocyte vitrification does not increase the risk of embryonic aneuploidy or diminish the implantation potential of blastocysts created after intracytoplasmic sperm injection: a novel, paired randomized controlled trial using DNA fingerprinting. Fertility and Sterility. 2012; 98:644-649. [PubMed: 22608316]

14. Blakeley P, et al. Defining the three cell lineages of the human blastocyst by single-cell RNA-seq. Development. 2015; 142:3151-3165. [PubMed: 26293300]

15. van der Maaten LJP, GE H. Visualizing High-Dimensional Data Using t-SNE. Journal of Machine Learning Research 9(Nov):2579-2605, 2008. Journal of Machine Learning Research. 2008; 9:2579-2605.

16. Hellebrekers DMEI, et al. PGD and heteroplasmic mitochondrial DNA point mutations: a systematic review estimating the chance of healthy offspring. Human Reproduction Update. 18:341-349. 
17. Samuels DC, Wonnapinij P, Chinnery PF. Preventing the transmission of pathogenic mitochondrial DNA mutations: can we achieve long-term benefits from germ-line gene transfer? Human Reproduction. 2013; 28:554-559. [PubMed: 23297368]

18. Facucho-Oliveira JM, John JC. The relationship between pluripotency and mitochondrial DNA proliferation during early embryo development and embryonic stem cell differentiation. Stem cell reviews. 2009; 5:140-158. [PubMed: 19521804]

19. Burgstaller JP, Johnston IG, Poulton J. Mitochondrial DNA disease and developmental implications for reproductive strategies. Mol Hum Reprod. 2015; 21:11-22. [PubMed: 25425607]

20. Hämäläinen RH, et al. Tissue- and cell-type-specific manifestations of heteroplasmic mtDNA $3243 \mathrm{~A}>\mathrm{G}$ mutation in human induced pluripotent stem cell-derived disease model. Proceedings of the National Academy of Sciences. 2013; 110:E3622-E3630.

21. Lee HS, et al. Rapid mitochondrial DNA segregation in primate preimplantation embryos precedes somatic and germline bottleneck. Cell Rep. 2012; 1:506-515. [PubMed: 22701816]

22. Treff NR, et al. Blastocyst preimplantation genetic diagnosis (PGD) of a mitochondrial DNA disorder. Fertility and Sterility. 2012; 98:1236-1240. [PubMed: 22921075]

23. Mitalipov S, Amato P, Parry S, Falk MJ. Limitations of Preimplantation Genetic Diagnosis for Mitochondrial DNA Diseases. Cell Reports. 2014; 7:935-937. [PubMed: 24856294]

24. Sallevelt SCEH, et al. Preimplantation genetic diagnosis in mitochondrial DNA disorders: challenge and success. Journal of Medical Genetics. 2013; 50:125-132. [PubMed: 23339111]

25. Steffann J, et al. Analysis of mtDNA variant segregation during early human embryonic development: a tool for successful NARP preimplantation diagnosis. Journal of Medical Genetics. 2006; 43:244-247. [PubMed: 16155197]

26. Herbert M, Kalleas D, Cooney D, Lamb M, Lister L. Meiosis and maternal aging: insights from aneuploid oocytes and trisomy births. Cold Spring Harb Perspect Biol. 2015; 7:a017970. [PubMed: 25833844]

27. Choudhary M, et al. Egg Sharing for Research: A Successful Outcome for Patients and Researchers. Cell Stem Cell. 2012; 10:239-240. [PubMed: 22385652]

28. HFEA Guidance on Payments for Donors. HFEA Code of Practice. Section 13http:// www.hfea.gov.uk/500.html

29. Hyslop L, et al. A Novel Isolator-Based System Promotes Viability of Human Embryos during Laboratory Processing. PLoS ONE. 2012; 7:e31010. [PubMed: 22393356]

30. Cutting R, Morroll D, Roberts SA, Pickering S, Rutherford A. Elective Single Embryo Transfer: Guidelines for Practice British Fertility Society and Association of Clinical Embryologists. Human Fertility. 2008; 11:131-146. [PubMed: 18766953]

31. Stephenson EL, Braude PR, Mason C. International community consensus standard for reporting derivation of human embryonic stem cell lines. Regenerative medicine. 2007; 2:349-362. [PubMed: 17635044]

32. Wells D, et al. Clinical utilisation of a rapid low-pass whole genome sequencing technique for the diagnosis of aneuploidy in human embryos prior to implantation. J Med Genet. 2014; 51:553-562. [PubMed: 25031024]

33. Kim D, et al. TopHat2: accurate alignment of transcriptomes in the presence of insertions, deletions and gene fusions. Genome Biology. 2013; 14:R36. [PubMed: 23618408]

34. Anders S, Pyl PT, Huber W. HTSeq--a Python framework to work with high-throughput sequencing data. Bioinformatics. 2015; 31:166-169. [PubMed: 25260700]

35. Robinson MD, McCarthy DJ, Smyth GK. edgeR: a Bioconductor package for differential expression analysis of digital gene expression data. Bioinformatics. 2010; 26:139-140. [PubMed: 19910308]

36. Love MI, Huber W, Anders S. Moderated estimation of fold change and dispersion for RNA-seq data with DESeq2. Genome Biol. 2014; 15:550. [PubMed: 25516281]

37. Taylor RW, Taylor GA, Durham SE, Turnbull DM. The determination of complete human mitochondrial DNA sequences in single cells: implications for the study of somatic mitochondrial DNA point mutations. Nucleic Acids Res. 2001; 29:E74-74. [PubMed: 11470889]

38. Andrews RM, et al. Reanalysis and revision of the Cambridge reference sequence for human mitochondrial DNA. Nat Genet. 1999; 23:147. [PubMed: 10508508] 
39. Greaves LC, et al. Clonal Expansion of Early to Mid-Life Mitochondrial DNA Point Mutations Drives Mitochondrial Dysfunction during Human Ageing. PLoS Genet. 2014; 10:e1004620. [PubMed: 25232829]

40. Wamaitha SE, et al. Gata6 potently initiates reprograming of pluripotent and differentiated cells to extraembryonic endoderm stem cells. Genes Dev. 2015; 29:1239-1255. [PubMed: 26109048]

41. Mortazavi A, Williams B, McCue K, Schaeffer L, Wold B. Mapping and quantifying mammalian transcriptomes by RNA-Seq. Nat Methods. 2008; 5:621-628. [PubMed: 18516045]

42. White HE, et al. Accurate detection and quantitation of heteroplasmic mitochondrial point mutations by pyrosequencing. Genetic testing. 2005; 9:190-199. [PubMed: 16225398] 
a Completion of meiosis

$1^{\text {st }}$ mitotic cell cycle

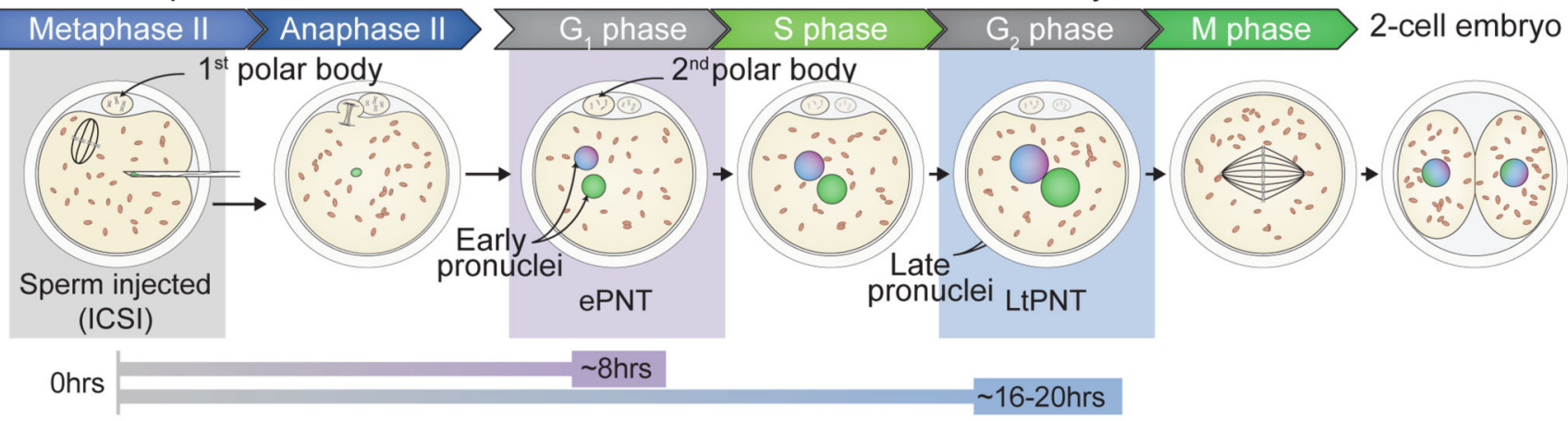

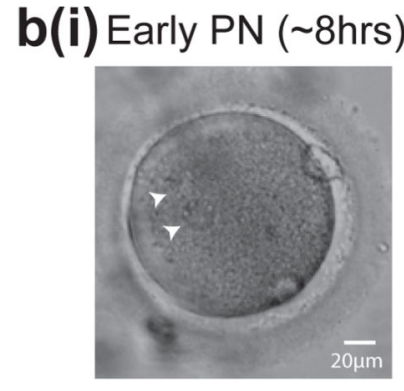

$$
\downarrow \begin{gathered}
+ \text { cytoskeletal } \\
\text { inhibitors } \\
+/- \text { sucrose }
\end{gathered}
$$

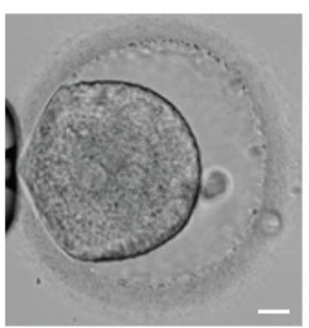

(ii) Enucleation
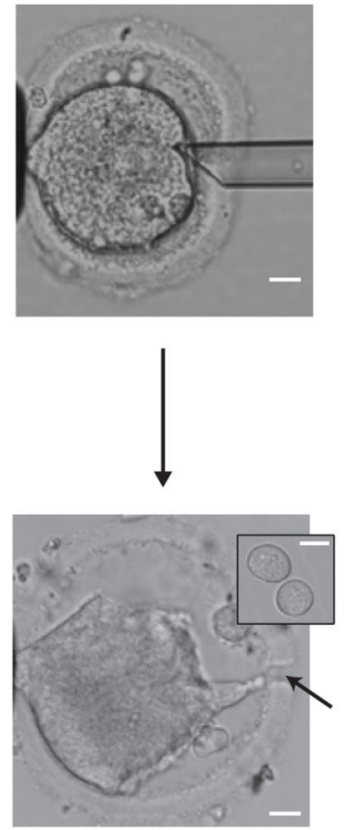

(iii) Fusion

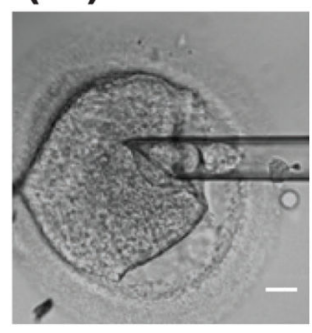

Excess cytoplasm removed

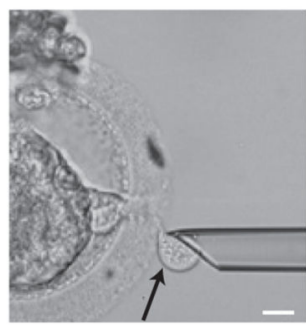

C

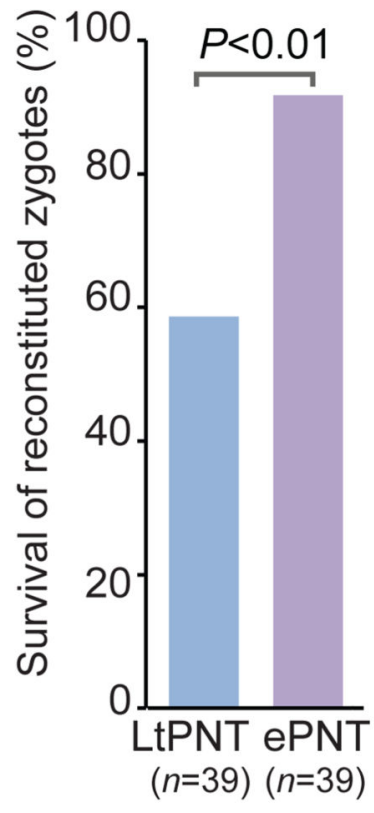

Figure 1. Early PNT promotes survival of normally fertilized zygotes following PNT.

a, Progression from MII arrest to completion of the 1st mitosis showing timings of early PNT (ePNT) and late PNT (LtPNT) b, Images show the steps involved in ePNT (i)

Arrowheads indicate the pronuclei (PN) (ii) Enucleation pipette inserted through a laserinduced opening in the zona pellucida (arrow, bottom panel). Lower panel, enucleated zygote (cytoplast). Inset shows two karyoplasts, each consisting of a single PN surrounded by a small amount of cytoplasm. (iii) Karyoplasts treated with HVJ-E and inserted under the zona pellucida. Lower panel, arrow indicates removal of excess cytoplasm (see Video 2). c, Survival of reconstituted ePNT and LtPNT zygotes $(P<0.01)$. Comparisons by $\chi^{2}$ test. 
a

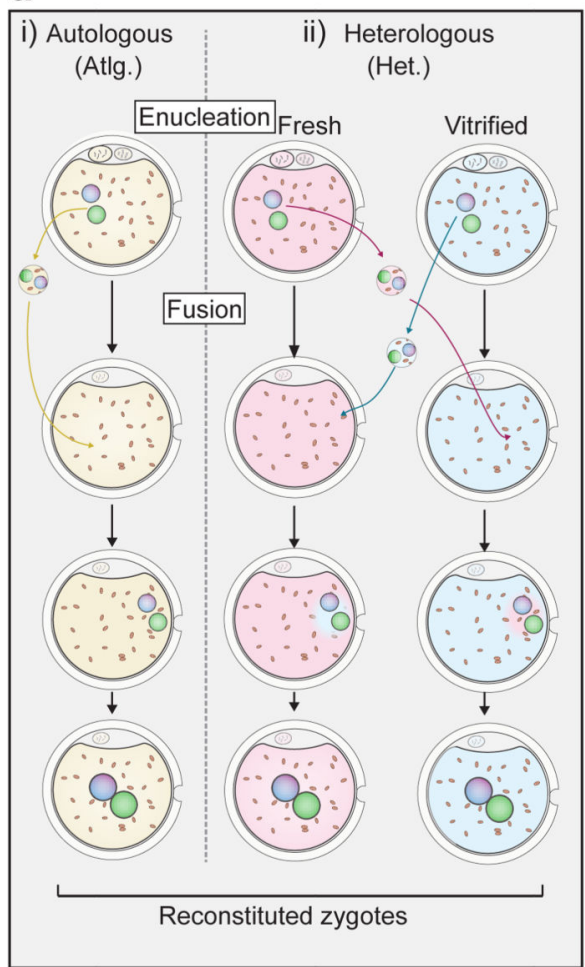

b

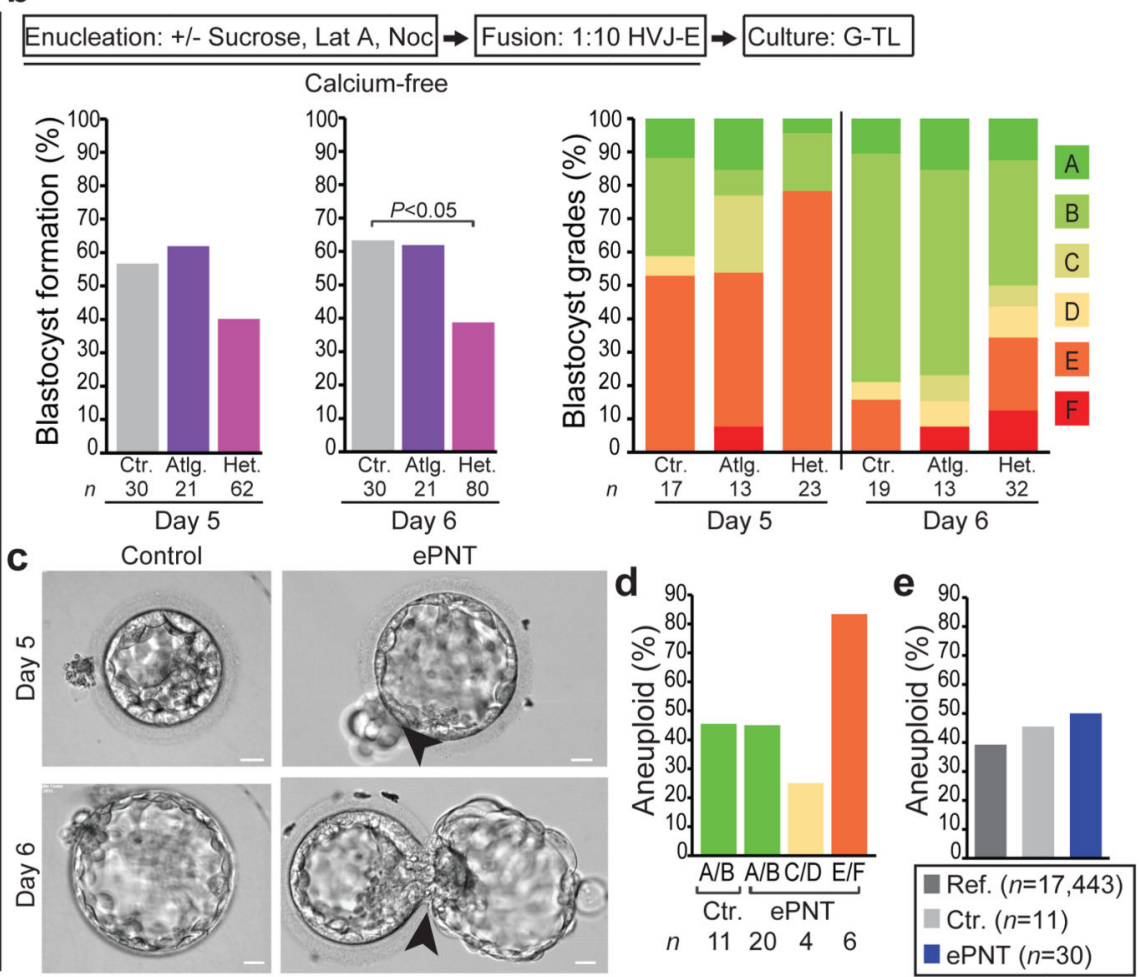

Figure 2. Blastocyst development following ePNT

a, Autologous (Atlg) ePNT (i) and Heterologous (Het) ePNT (ii). b, Blastocyst formation and quality (series II). Zygotes ( $n=131$ from 30 donors) were either unmanipulated (Ctr, $n=30)$; or used for Atlg ( $n=21)$, or Het $(n=80)$ ePNT. Het ePNT blastocyst formation was reduced compared with $\operatorname{Ctr}\left(P<0.05 ; \chi^{2}\right.$ test $)$. Blastocyst quality is similar between the three groups (not significant; Fisher's exact test). c, Images showing good quality control and ePNT blastocysts. Arrowheads, ePNT blastocyst hatching through laser-induced opening in the zona pellucida. Scale bars, $20 \mu \mathrm{m}$. d, Aneuploidy in cells sampled from blastocysts (grades A-F) detected by array-CGH blind to sample origin ( $n=$ blastocysts; not significant, Fisher's exact test). e, Aneuploidy in controls and ePNT blastocysts not significantly different from a reference population (Ref) of IVF blastocysts $\left(\chi^{2}\right.$ test). (Source data available online for part b). 

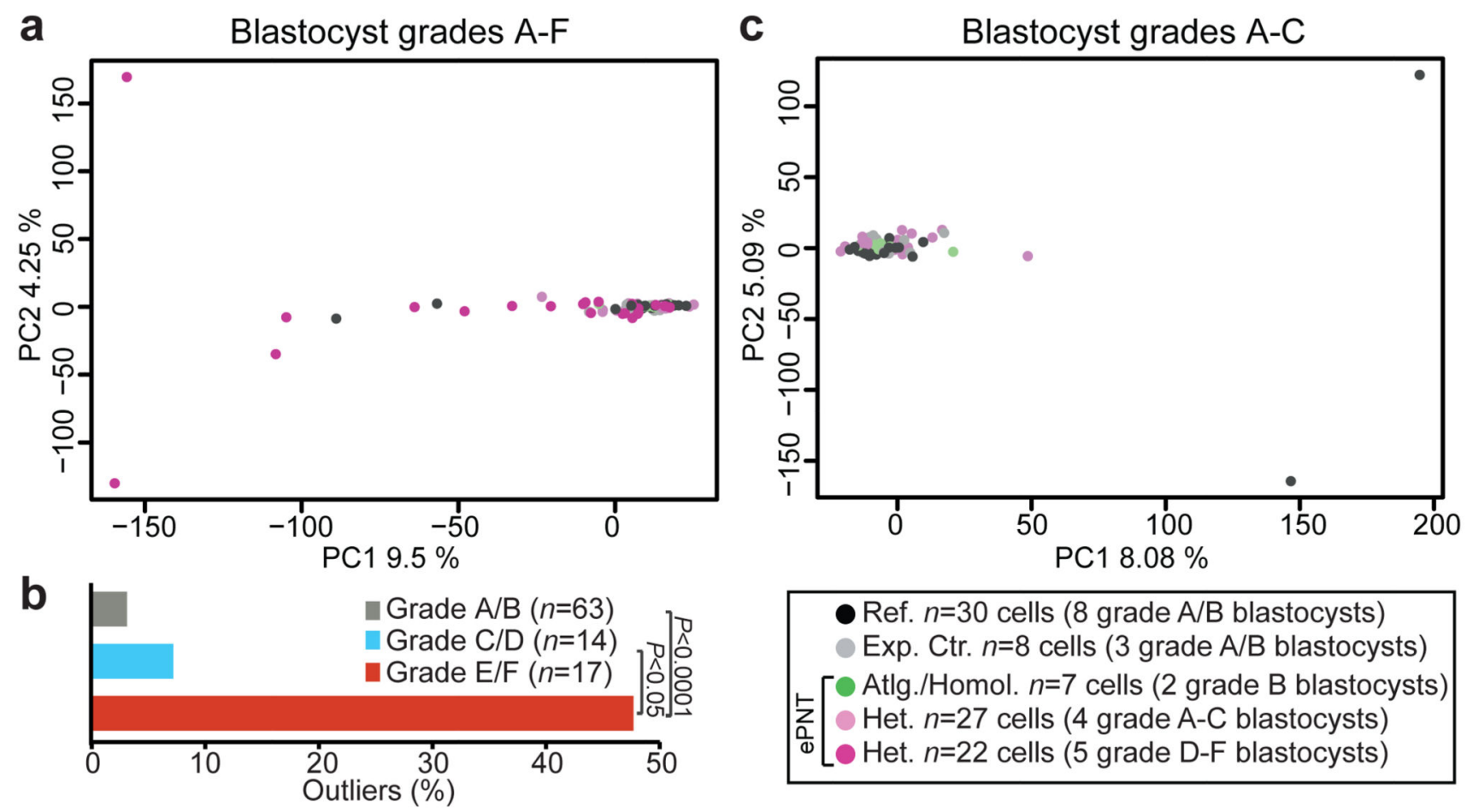

Figure 3. Gene expression patterns in unmanipulated controls and ePNT blastocysts.

a, Principal Component Analysis (PCA) of single cell RNA-seq data showing global gene expression profiles in a reference population ( $n=30$ samples; 8 blastocysts), experimental controls ( $n=8$ samples; 3 blastocysts) and ePNT blastocysts ( $n=56$ samples; 11 blastocysts). Each data point represents a single cell. ePNT blastocysts include heterologous (Het) autologous (Atlg) and homologous (Homol) transfers (Extended Data Fig. 5b). The Het group is subdivided according to blastocyst grade. b, Graph shows an increased percentage of outliers in poor quality blastocysts ( $P$ values shown; Fisher's exact test). c, PCA of single cell RNA-seq data comparing control samples with good quality ePNT blastocysts. PCA was performed blind to sample origin. The number of samples and blastocysts are shown. (Data deposited to Gene Expression Omnibus GSE76284). 
a

Freshly harvested Vitrified
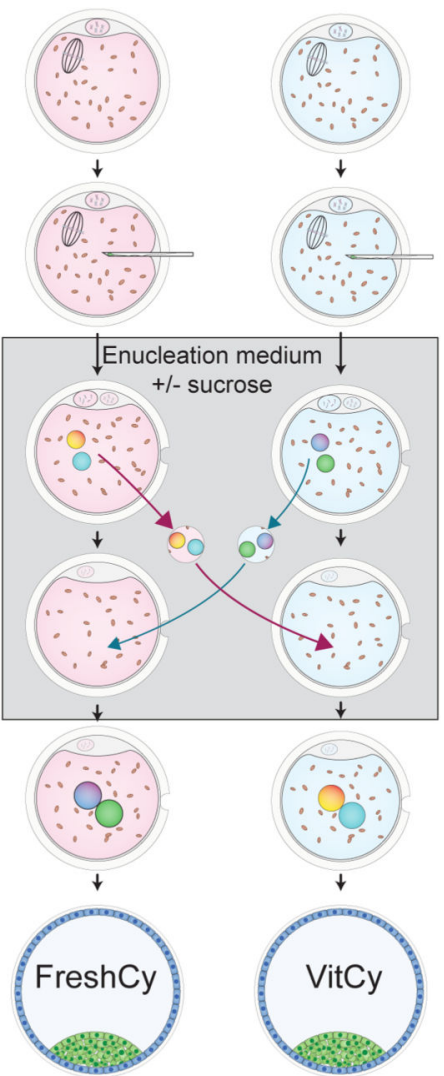

Fresh cytoplast/ Vitrified karyoplast

d

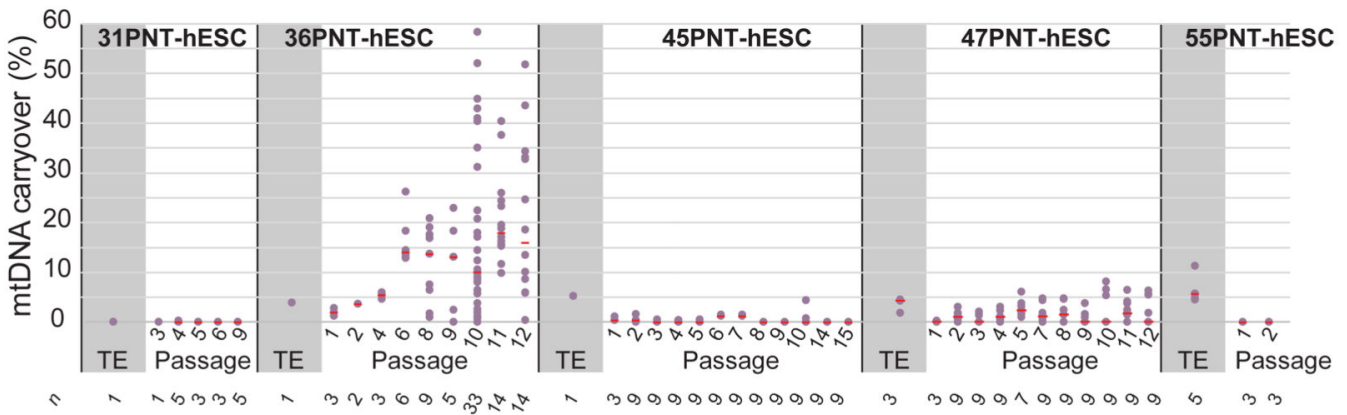

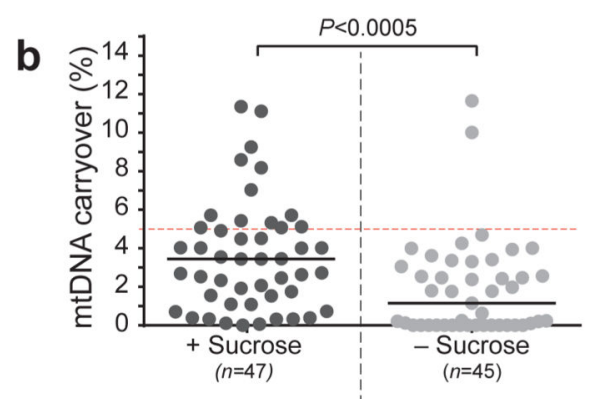

C
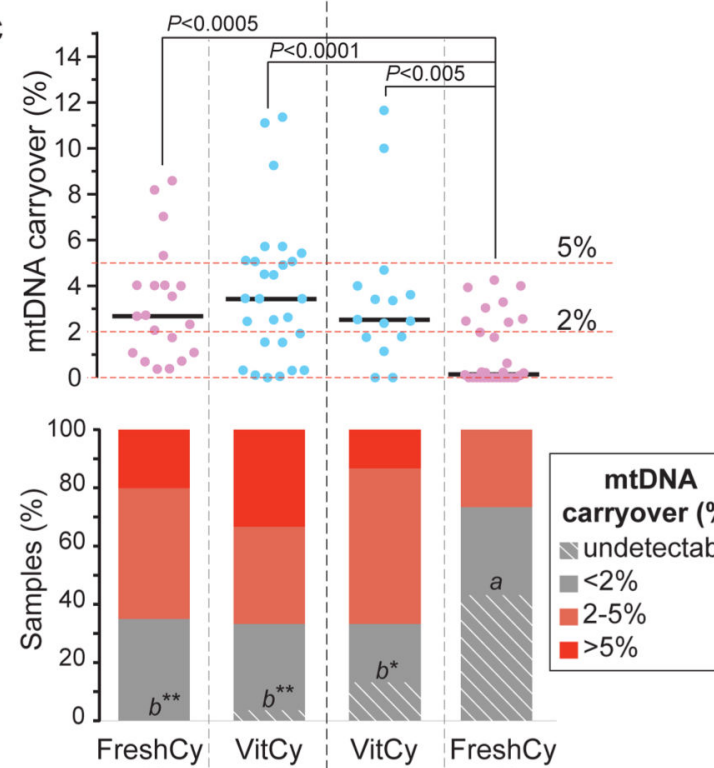

carryover (\%)

sundetectable $-<2 \%$

$-2-5 \%$

$>5 \%$

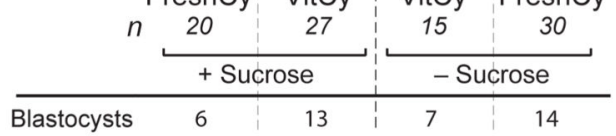

Blastocysts

Figure 4. Carryover of karyoplast mtDNA in ePNT blastocysts

a, Reciprocal ePNT between zygotes from fresh and vitrified oocytes, enucleated in the presence or absence of sucrose resulting in four experimental groups: FreshCy \pm sucrose; VitCy \pm sucrose. $\mathbf{b}$, MtDNA carryover in day 6 blastocysts arising from ePNT in the presence or absence of sucrose; horizontal lines represent means $(P<0.0005$; two-sided Mann-Whitney U test). c, MtDNA carryover in day 6 blastocysts (FreshCy and VitCy) following ePNT in the presence or absence of sucrose; horizontal lines represent medians $(P$ vales shown, two-sided Mann-Whitney U test). Stacked graph showing the percentage of 
samples with specified levels of heteroplasmy. Groups with different letters are significantly different (* $P<0.05 ; * * P<0.01 ; \chi^{2}$ test). b,c Data points represent means of 2-3 technical replicates; $n=$ number of samples, numbers of blastocysts as shown in c. d, MtDNA carryover in PNT-hESC lines ( $n=5)$. Data points represent $2-3$ technical replicates. Median value (red) for each passage in which $>1$ sample was tested; $n=$ number of samples tested for each passage. (Source data files available online for b-d). 